Atmos. Chem. Phys., 17, 12269-12302, 2017

https://doi.org/10.5194/acp-17-12269-2017

(C) Author(s) 2017. This work is distributed under

the Creative Commons Attribution 3.0 License.

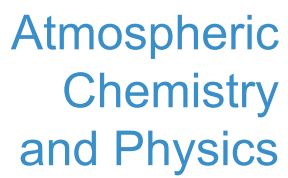

(c) (P)

\title{
Reconciling differences in stratospheric ozone composites
}

\author{
William T. Ball ${ }^{1,2}$, Justin Alsing ${ }^{3,4}$, Daniel J. Mortlock ${ }^{4,5,6}$, Eugene V. Rozanov ${ }^{1,2}$, Fiona Tummon ${ }^{1}$, and \\ Joanna D. Haigh ${ }^{4,7}$ \\ ${ }^{1}$ Institute for Atmospheric and Climate Science, Swiss Federal Institute of Technology Zurich, \\ Universitaetstrasse 16, CHN, 8092 Zurich, Switzerland \\ ${ }^{2}$ Physikalisch-Meteorologisches Observatorium Davos World Radiation Centre, Dorfstrasse 33, \\ 7260 Davos Dorf, Switzerland \\ ${ }^{3}$ Center for Computational Astrophysics, Flatiron Institute, 162 5th Ave, New York, NY 10010, USA \\ ${ }^{4}$ Physics Department, Blackett Laboratory, Imperial College London, SW7 2AZ London, UK \\ ${ }^{5}$ Department of Mathematics, Imperial College London, SW7 2AZ London, UK \\ ${ }^{6}$ Department of Astronomy, Stockholms universitet, 10691 Stockholm, Sweden \\ ${ }^{7}$ Grantham Institute - Climate Change and the Environment, Imperial College London, SW7 2AZ London, UK \\ Correspondence to: William T. Ball (william.ball@env.ethz.ch)
}

Received: 24 February 2017 - Discussion started: 15 March 2017

Revised: 28 July 2017 - Accepted: 22 August 2017 - Published: 16 October 2017

\begin{abstract}
Observations of stratospheric ozone from multiple instruments now span three decades; combining these into composite datasets allows long-term ozone trends to be estimated. Recently, several ozone composites have been published, but trends disagree by latitude and altitude, even between composites built upon the same instrument data. We confirm that the main causes of differences in decadal trend estimates lie in (i) steps in the composite time series when the instrument source data changes and (ii) artificial sub-decadal trends in the underlying instrument data. These artefacts introduce features that can alias with regressors in multiple linear regression (MLR) analysis; both can lead to inaccurate trend estimates. Here, we aim to remove these artefacts using Bayesian methods to infer the underlying ozone time series from a set of composites by building a joint-likelihood function using a Gaussian-mixture density to model outliers introduced by data artefacts, together with a data-driven prior on ozone variability that incorporates knowledge of problems during instrument operation. We apply this Bayesian self-calibration approach to stratospheric ozone in $10^{\circ}$ bands from $60^{\circ} \mathrm{S}$ to $60^{\circ} \mathrm{N}$ and from 46 to $1 \mathrm{hPa}(\sim 21-48 \mathrm{~km})$ for 1985-2012. There are two main outcomes: (i) we independently identify and confirm many of the data problems previously identified, but which remain unaccounted for in existing composites; (ii) we construct an ozone composite, with uncertainties, that is free from most of these problems - we
\end{abstract}

call this the BAyeSian Integrated and Consolidated (BASIC) composite. To analyse the new BASIC composite, we use dynamical linear modelling (DLM), which provides a more robust estimate of long-term changes through Bayesian inference than MLR. BASIC and DLM, together, provide a step forward in improving estimates of decadal trends. Our results indicate a significant recovery of ozone since 1998 in the upper stratosphere, of both northern and southern midlatitudes, in all four composites analysed, and particularly in the BASIC composite. The BASIC results also show no hemispheric difference in the recovery at midlatitudes, in contrast to an apparent feature that is present, but not consistent, in the four composites. Our overall conclusion is that it is possible to effectively combine different ozone composites and account for artefacts and drifts, and that this leads to a clear and significant result that upper stratospheric ozone levels have increased since 1998, following an earlier decline.

\section{Introduction}

The ozone layer in the stratosphere protects the Earth's biosphere from harmful solar ultraviolet (UV) radiation. The use of ozone-depleting substances (ODSs), including chlorofluorocarbons (CFCs), led to a decline in ozone globally over the latter half of the 20th century (Johnston, 1971; 
Crutzen, 1971; Molina and Rowland, 1974), particularly in the polar regions (WMO, 2011, 2014). The implementation of the Montreal Protocol (MP), which banned the use of most ODSs, has halted this decline, and in some regions there has been a recovery in total column ozone (Solomon et al., 2016). However, there is large uncertainty in the sign and magnitude of recent trends depending on altitude and latitude, and a clear signal is difficult to determine (Harris et al., 2015).

Ozone responds to forcings from below, e.g. injections of aerosols from volcanoes (Robock, 2000) or wave activity from the troposphere (Kidston et al., 2015), and from above, e.g. from solar sources such as UV radiation (Haigh, 1994) and particles (Funke et al., 2011; Mironova et al., 2015). In order to quantify and understand the variability forced by a particular driver, and long-term trends in ozone - not just in terms of the total column ozone (TCO) but also resolved vertical profiles - observations spanning multiple decades are needed. Such a dataset can only be provided by combining data from multiple sources (Harris et al., 2015; Tummon et al., 2015). The method used to combine the data needs to consider different inherent attributes, the most important of which include temporal resolution, vertical and horizontal spatial resolution (Kramarova et al., 2013a), time of day and geolocation of observations (Sofieva et al., 2014), absolute calibration (Frith et al., 2014), and stability estimates and instrument uncertainty (DeLand et al., 2012). All of these factors, if not well accounted for, can introduce additional (artificial) trends, uncertainties, and errors, which may leak into statistical analyses of decadal trends (Harris et al., 2015; Tummon et al., 2015) and estimates of the magnitude of the response to drivers such as the Sun (Maycock et al., 2016). This can lead to conflicting results from different datasets (WMO, 2014).

Observational records of atmospheric ozone began with ground-based observations in 1921 (Staehelin et al., 1998) and were joined by satellite observations in the 1960s (Krueger et al., 1980). These records are an invaluable tool to understand not only the long-term trends in ozone but also how the middle atmosphere operates. Ground-based observations have the advantage of being longer records and can be recalibrated on a continuous basis, but they are point-source observations and thus cannot account for large differences in ozone concentration and variability with latitude and longitude. The introduction of satellite observations has allowed for near-global, continuous observations over many decades but has the disadvantages of typically only operating for a limited number of years and being subject to space-based degradation.

Creating an accurate record of stratospheric ozone profiles is a non-trivial task and much work has been done at every stage, from design, construction, and operation during flight, to post-processing and combining datasets into composites (Kyrölä et al., 2013; McPeters et al., 2013; Sofieva et al., 2013; Sioris et al., 2014; Froidevaux et al., 2015; Davis et al., 2016). Recently, several composites were published by mul- tiple groups in connection with the SI2N initiative (SPARC (Stratosphere-troposphere Processes And their Role in Climate)/IO3C (International Ozone Commission)/IGACO-O3 (Integrated Global Atmospheric Chemistry Observations Ozone)/NDACC (Network for the Detection of Atmospheric Composition Change)) (Tummon et al., 2015). Nevertheless, even when problems are flagged and uncertainties are minimized, the fact that different composites can lead to trend estimates that differ by more than their uncertainties (e.g. Fig. 6 of Harris et al., 2015 and Fig. 8 of Tummon et al., 2015) means that at least one, if not all, are insufficiently stable during some periods to provide a robust estimate of changes in ozone throughout the stratosphere. Tummon et al. (2015) further notes that the choice of instruments to merge has more impact on trends than the merging technique used, that the construction approach needs careful consideration of the method used to avoid contaminating trends with artefacts, and that so far it has not been possible to remove biases from any individual, vertically resolved dataset.

Despite these difficulties, it is possible to account for many of these problems. There is common information within all the composites, e.g. the annual variability is similar in most composites (Tummon et al., 2015), and the differences between composite datasets due to the issues listed above should, in principle, point to where potential artefacts such as steps and drifts are located in time and by latitude and altitude. This can be especially effective in the case of an unexpected or erroneous change occurring in one dataset, which is absent in all the others. Once the instrument or composite at fault is identified, there is the possibility of flagging, removing, or rectifying an error, and confidence in applying a correction increases if the deviation or fault can be linked to a known issue. Thus, together with this prior knowledge and an unbiased uncertainty estimate, one can evaluate the likelihood of an observation being correct or, indeed, estimate the most likely value.

Our goal here is to provide a technique whereby the most likely ozone variability throughout the stratosphere can be identified by using the information embedded within multiple datasets simultaneously. The natural approach with which to tackle such a problem is using Bayesian inference (Cox, 1946; Lee et al., 2005; Arnold et al., 2007). In adopting a Bayesian approach, we develop a detailed probabilistic model for the (multiple) datasets, carefully allowing for outliers and accounting for all knowledge (and ignorance) of measurement uncertainties and any known problems during instrument operation. Additionally, by incorporating (datadriven) prior information about the underlying ozone variability, we are able to identify - using only the data and knowledge of the instruments - where some datasets are systematically biased due to measurement artefacts whilst others are consistent with the anticipated month-to-month variability. In this way, our approach combines the multiple datasets in such a way that they "self-calibrate" each other, resulting in a single ozone time series that is cleaned of many of the 
artefacts affecting any individual dataset (although if a problem is common to all datasets, it cannot be identified).

This paper has three main parts. In the first part (Sect. 2), we introduce the composite datasets we use (Sect. 2.1) by explicitly presenting the problems we will later attempt to fix. Ozone composites have been updated since important intercomparison papers by Harris et al. (2015) and Tummon et al. (2015), so our results cannot be directly compared with theirs; we briefly present some of these differences (Sect. 2.2). The ozone composites, described in Sect. 2, form a good starting point from which to combine information and account for differences, since the effort put into producing them already considers and accounts for many instrument and observational issues. However, some remaining problems are clear in the composites. In the second part, we present the Bayesian method to self-correct the ozone composites (Sect. 3), construct uncertainty estimates (Sect. 3.1), form the Gaussian-mixture likelihood (Sect. 3.2), develop transition priors to estimate how ozone is expected to vary on monthly timescales (Sect. 3.3), and discuss how we include additional prior information that we have available (Sect. 3.1). We call this combined set of steps and algorithms the BAyeSian Integrated and Consolidated (BASIC) approach. The resulting BASIC composite time series are presented and compared with the composites in Sect. 4.2 and 4.3. In the final part (Sect. 5), we primarily use dynamical linear modelling (DLM) to evaluate long-term trends (Sect. 5.2), although we compare our results with multiple linear regression (MLR) analysis, and present our results for ozone changes over the 1985-2012 period in Sect. 5.3. We conclude in Sect. 6.

\section{Data}

\subsection{Ozone composites}

The SI2N project promoted seven ozone composites of satellite observations, summarized in Tummon et al. (2015), along with detailed comparisons that were expanded upon by Harris et al. (2015). Three of the datasets, named SAGEGOMOS1 (Kyrölä et al., 2013), SAGE-GOMOS2 (Tummon et al., 2015), and SAGE-OSIRIS (Adams et al., 2014) in Tummon et al. (2015), have more data missing than the others $\left(\sim 57 \%\right.$ for $1985-2012$ for $\left.20^{\circ} \mathrm{S}-20^{\circ} \mathrm{N}\right)$, so we do not consider them in our analysis. Two of the remaining composites have the SAGE-II instrument (Stratospheric Aerosol and Gas Experiment II) (Damadeo et al., 2013) as a backbone: GOZCARDS (Global OZone Chemistry And Related Datasets for the Stratosphere; Froidevaux et al., 2015) and SWOOSH (Stratospheric Water and Ozone Satellite Homogenized; Davis et al., 2016); we will refer to this pair of composites as "SAGE-based". The other two "SBUVbased" composites we consider use the suite of SBUVtype (solar backscatter ultraviolet) instruments: SBUV-MOD
(SBUV version 8.6 merged ozone data set; Frith et al., 2014) and SBUV-MER (SBUV Merged Cohesive; Wild and Long, 2017). By using only two pairs of composites containing approximately equal weighting, we partly avoid the issue of biasing results to SAGE-based composites, a concern raised in the analysis of Harris et al. (2015) (however, see Appendix Sect. A5.2).

We consider zonal mean, monthly mean ozone over the 28-year period, January 1985-December 2012, covered by all datasets. While the correction method we present later (Sect. 3) could, in principle, be used to deal with data gaps at higher latitudes, we limit our latitude range to 12 bands of $10^{\circ}$ over $60^{\circ} \mathrm{S}-60^{\circ} \mathrm{N}$. We limit the pressure range to 11 levels from 46 to $1 \mathrm{hPa}(\sim 21-48 \mathrm{~km})$ to avoid issues of large diurnal variations at higher altitudes, and because the vertically resolved SBUV data are not available at lower altitudes (i.e. at higher pressures); note, however, that some diurnal variability exists down to $5 \mathrm{hPa}$. In order to treat each composite fairly, we interpolate all four onto the GOZCARDS pressure-latitude grid since this grid has the lowest resolution of the four (though the instruments themselves have a higher resolution); a visualization of the original grids are shown in Fig. A1 in the Appendix. All considered composites have data available for more than $80 \%$ of all months at most latitudes. Finally, for this work, we are interested in relative variability and trends, so we shift absolute values to agree with the mean of SWOOSH from August 2005 to December 2012 when the Aura/MLS instrument is used; during this period, all the composites show remarkably good agreement on annual and multi-year timescales, and regression coefficients using multiple linear regression (see Sect. 5.1) are similar at all pressure levels and latitudes (not shown). This is important since a common reference period we trust improves the ability for the BASIC approach to estimate relative changes and reduces uncertainties.

The ozone instrument data and composites are already extensively detailed and discussed in several recent papers as listed above, e.g. Tummon et al. (2015) and Harris et al. (2015); we recommend that interested readers consult these papers, which also include an exhaustive list of references to individual instruments. We will discuss relevant points of interest regarding each composite in the discussion that follows below.

\subsection{Inconsistencies between composites}

To determine why decadal trends from the various composites are different requires an understanding of how they have been constructed with satellite instrument data from multiple sources. We present a visual reference guide for the four composites in Fig. 1. Here, we show the timeline of instruments used to construct the SAGE-based data in the middle and SBUV below. The colour coding for the four datasets (GOZCARDS in dark blue, SWOOSH in light blue, SBUV-MOD in red, and SBUV-MER in yellow) will be used 


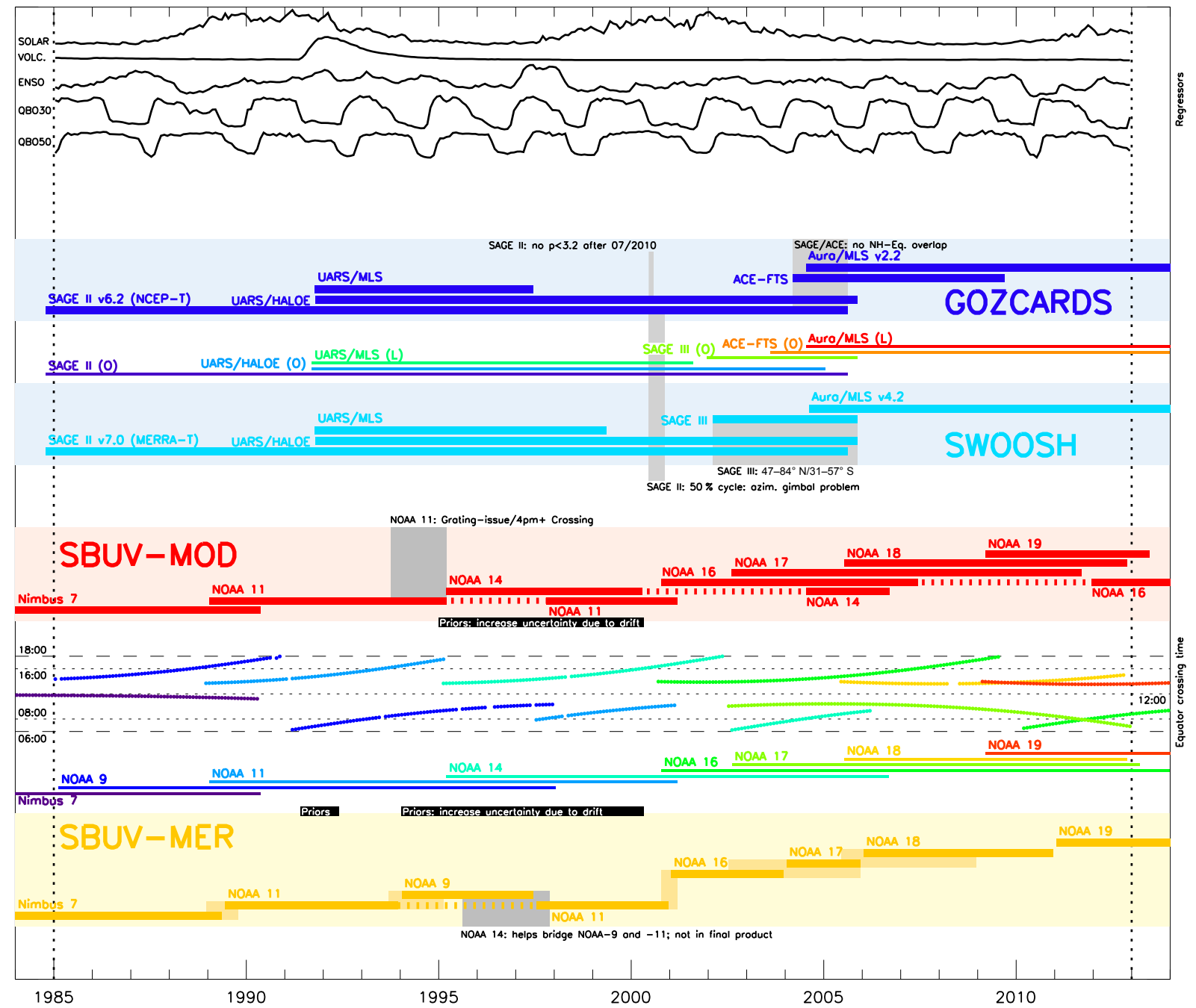

Figure 1. A guide to the regression indices used in the trend analysis (upper third) and instrument data used to construct SAGE-based (middle third: GOZCARDS, dark blue; SWOOSH, light blue) and SBUV-based (lower third: SBUV-MOD, red; SBUV-MER, yellow) composites. Shading at SBUV-MER instrument changes indicates periods used to determine differences in annual variability and applying bias corrections between instruments. The full periods of instrument operation for datasets in these pairs are shown with multiple colours between the composites. Where SBUV data are not used for an interval, dashed lines replace solid. Between the SBUV composites, the local time of Equator crossing is shown. Where relevant, version numbers are given with instrument names; "O" and " $L$ " indicate the satellite was a limb viewer or occultation-based instrument; SBUV instruments are all nadir viewing. Grey shading with black text highlights periods discussed in the article. Periods specifically flagged to increase the SBUV uncertainty estimates in the BASIC approach are labelled black with white text.

throughout the paper. The operating periods of all the instrument datasets used for either SWOOSH or GOZCARDS are presented as a spectrum of colours between them; the same is done for the SBUV composites, where we additionally show information related to the time of day at which Equator crossings occur, which will be important later. Instrument names are given near the start of their operation period. Various comments and grey shadings litter the plot; these mark points to be aware of and some of these are discussed later.

\subsubsection{SBUV-based composites}

The two SBUV composites are built in two different ways: SBUV-MER uses overlapping time series (shading in Fig. 1) to calculate offsets (calibration biases) and differences in seasonal and diurnal variation, but only a single dataset is used without averaging overlapping periods; SBUV-MOD also accounts for offsets, but then overlapping data are averaged. SBUV-MOD relies on the instrument-to-instrument calibration done at the wavelength level within the version 8.6 al- 

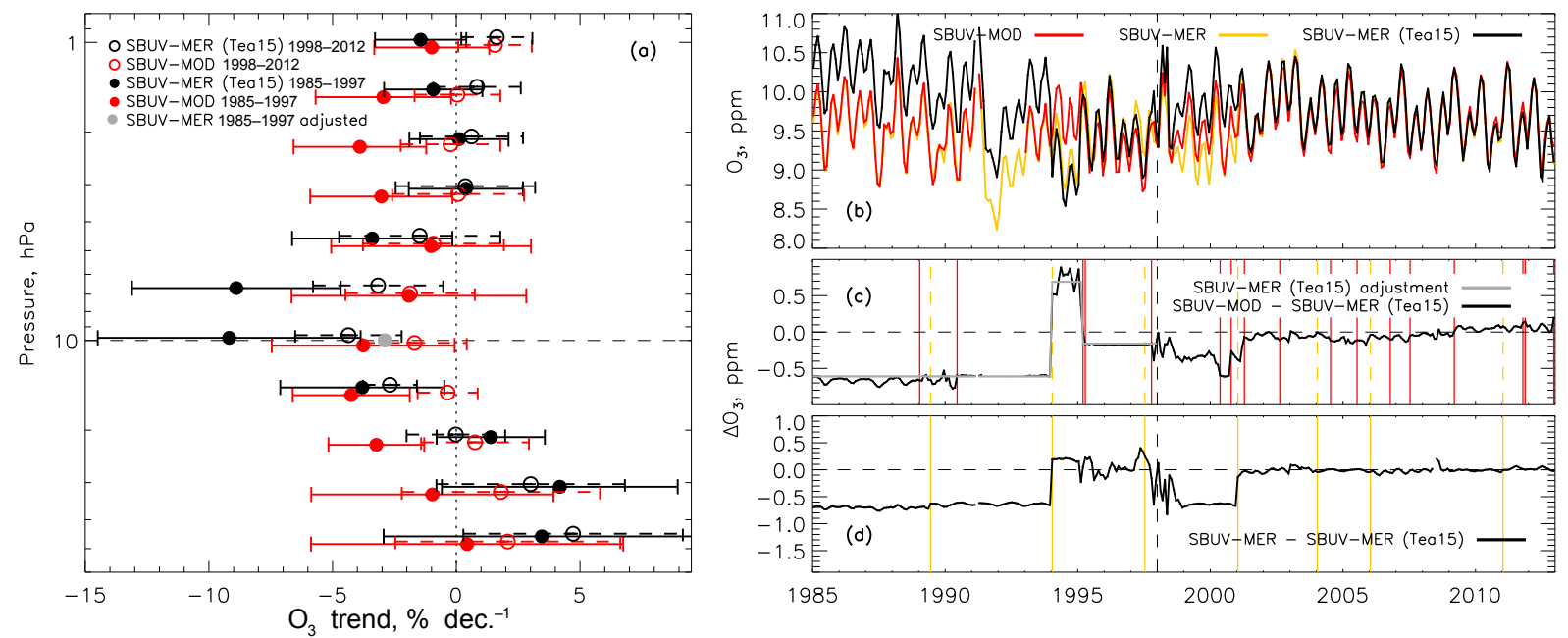

Figure 2. (a) The equatorial $\left(20^{\circ} \mathrm{S}-20^{\circ} \mathrm{N}\right)$ decadal ozone MLR trend profiles for the SBUV-MER version used by Tummon et al. (2015) ("Tea15"; black) and SBUV-MOD (red). Dots and solid error bars represent the 1985-1997 trends, and open circles and dashed error bars the 1998-2012 period. A single grey dot is plotted at $10 \mathrm{hPa}$, which follows an adjustment to SBUV-MER as shown in panel (c) as a grey line. (b) The ozone composite time series for SBUV-MER (Tea15) (black), SBUV-MER (yellow) and SBUV-MOD (red) at $10 \mathrm{hPa}$, all shifted to the July 2005-2012 mean of SWOOSH. (c) The difference between the SBUV-MOD and MER (Tea15) time series in panel (b); the grey line prior to 1998 is a correction applied to SBUV-MER (Tea15) to produce the grey dot in panel (a). (d) The difference between SBUV-MER (Tea15) and SBUV-MER. The vertical dashed line in panels (b-d) indicates 1 January 1998, which delimits the two periods considered in the MLR results in panel (a). Error bars are $2 \sigma$.

gorithm for absolute calibration (i.e. no additional offsets are applied before averaging).

The SBUV-based composites use only instruments with the same design and are the longest single-instrument-type composites available. Both use the same NOAA and Nimbus space-based platforms, though not always at the same time, except that SBUV-MER uses NOAA-9 observations between 1994 and 1997 to increase global coverage and bridge the gap in NOAA-11 (Fig. 1), which is an update to SBUVMER that differs from the previous version considered by Tummon et al. (2015) (see below); SBUV-MER also uses NOAA-14 as a backbone to connect biases in NOAA-9 and -11 , but the NOAA-14 data are not used in the final product. The SBUV instruments infer profile ozone in units of parts per million ( $\mathrm{ppm})$ volume mixing ratio from measurements of back-scattered UV radiation at wavelengths shorter than $300 \mathrm{~nm}$ in a downward, nadir viewing system, which is fundamentally different from the limb/occultation instruments used in the SAGE-based composites; the SBUV instruments are optimized to low stray light and high signal-to-noise radiance measurements, with an estimated accuracy of 1-2 DU at solar zenith angles up to $70^{\circ}$ (McPeters et al., 2013). Despite being constructed with essentially the same instrument data, the two datasets show differences in estimated decadal trends (Harris et al., 2015; Tummon et al., 2015).

In Fig. 2a, we recreate the SBUV-MOD and SBUV-MER 1985-1997 (dots and solid lines) and 1998-2012 (circles and dashed lines) linear decadal ozone trend estimates from MLR (Sect. 5.1) for the equatorial regions $20^{\circ} \mathrm{S}-20^{\circ} \mathrm{N}$ as in Figs. 5 and 6 of Harris et al. (2015) and Fig. 8 of Tummon et al. (2015). SBUV-MER has seen revisions since it was used in Harris et al. (2015) and Tummon et al. (2015), so we use the version in those publications to make clear why previous analyses of the SBUV composites differ (labelled "Tea15"); after this section, we only consider the latest update. The two composites show good agreement over the 1998-2012 period in both mean value and profile shape. The earlier period shows different vertical structure; at $10 \mathrm{hPa}$, the mean values disagree by more than $5 \%$ per decade (the $10 \mathrm{hPa}$ level is indicated by the horizontal dashed line). The reason for this becomes obvious when we plot the absolute, and differences of, the time series at $10 \mathrm{hPa}$ in Fig. $2 \mathrm{~b}$ and c, respectively. Prior to 2002, the difference between SBUV-MER (Tea15) and SBUV-MOD can be almost as large as the annual variability. Figure $2 \mathrm{c}$ reveals that these are caused by steps, of which the two largest occur in January 1994 and FebruaryApril 1995. We plot coloured vertical lines when instruments in either composite change (yellow for SBUV-MER; red for SBUV-MOD), which immediately reveals that these jumps are related to offsets in instrument data: the first occurred in SBUV-MER; the second in SBUV-MOD. To prove it is these steps that cause the difference in the pre-1998 trend estimated at $10 \mathrm{hPa}$ in Fig. 2a, we simply subtract the grey curve indicated in Fig. 2c from SBUV-MER (Tea15) and the mean MLR estimate for the trend is indicated as a grey dot in Fig. 2a, now very close to SBUV-MOD. We note that this subtraction is not intended to indicate that SBUV-MOD is 
correct but is a simple test to understand why the trends differ.

Figure 2d shows the difference between SBUV-MER (Tea15) and the updated version, which shows many of the offsets relative to SBUV-MOD in Fig. 2c have been removed. However, artefacts still remain in the newer version with respect to SBUV-MOD, and we find that they are not confined just to the altitude and latitude range shown in these plots. Ultimately, the remaining differences will lead to the divergent trend estimates. We return to this in Sect. 4.3; further discussion on the SBUV composites is provided in Sect. A1.

\subsubsection{SAGE-based composites}

While constructed by two separate teams, GOZCARDS (Froidevaux et al., 2015) and SWOOSH (Davis et al., 2016) are similar for two main reasons: (i) the longest single instrument record used is SAGE-II (1984-2005) and this acts as the absolute reference level in both datasets; and (ii) they are constructed from limb viewers and occultation satellites (identified as "L" and "O" in Fig. 1), meaning they differ in operation from the SBUV nadir viewers. Occultation satellites measure ozone by looking at the disk of the rising or setting Sun though the atmosphere (SAGE-II uses the UV and visible, while, e.g. HALOE and ACE-FTS use infrared wavelengths); this makes their vertical profile resolution higher but at the expense of only observing 15 profiles per day. Limb sounders observe thermal emission in the infrared or microwave as volume mixing ratio on pressure levels and can observe thousands of profiles each day. The composites differ in several ways, the most relevant of which are (i) they use different data screening and preprocessing; (ii) data from the same satellites are used for different periods and/or spatial regions; (iii) SWOOSH contains SAGE-III data and not ACEFTS observations, and GOZCARDS vice versa (see Fig. 1); and (iv) GOZCARDS (v1.0, used here) uses SAGE-II version 6.2, while SWOOSH uses version 7.0 - this innocuous difference has consequences for the trends (and solar signal analysis; not shown) that we will elaborate on in the following.

Because SAGE-II observes ozone number density, knowledge of local temperature is needed to convert to volume mixing ratio. GOZCARDS uses SAGE-II v6.2, and SWOOSH SAGE-II v7.0; the former uses NCEP reanalysis temperatures while the latter uses the MERRA reanalysis (see Damadeo et al., 2013 and references within). It has been noted by McLinden et al. (2009), and confirmed by Maycock et al. (2016), that the NCEP temperature data contain spurious trends. The fact that the trend is not visible in SBUV data (Sect. 4.3) further supports this. The impact of the different versions of SAGE-II within the SAGE-based composites is shown in Fig. 3. We note that, as for SBUV-MER, the current SWOOSH release has changed with respect to the aforementioned publications. Therefore, we again initially show results from the earlier version (2.1) in red (again designated
"Tea15"); following this discussion we will not refer to this version again. Figure 3 a shows the equatorial $\left(20^{\circ} \mathrm{S}-20^{\circ} \mathrm{N}\right)$ decadal ozone trends similar to Fig. 2 extracted from GOZCARDS and SWOOSH (Tea15) using MLR for two periods: 1985-1997 (dots and solid lines) and 1998-2012 (circles and dashed lines). We see that for 1998-2012, except at 4.6 and $6.8 \mathrm{hPa}$, the two mean profiles agree well. However, for 1985-1997 above $5 \mathrm{hPa}$, the ozone profiles show very large differences. To clarify why, in Fig. 3b, we plot their $2.2 \mathrm{hPa}$ time series and their difference in Fig. 3c; the vertical dashed line indicates where the two periods considered in Fig. 3a are delimited. After 1991, both composites show similar long-term variability, though there are clearly sub-periods containing different scatter characteristics, and which change between instrument periods (vertical coloured lines), thus indicating a relationship to either different preprocessing or instrument usage. Between 1985 and 1991, GOZCARDS is lower than SWOOSH, and there appears to be an approximately linear increase over this period. Similar to the approach taken for SBUV-MER in Fig. 2, correcting the 1985-1991 period with a simple linear trend line (grey in Fig. 3c) leads to very good agreement with SWOOSH (Tea15) in Fig. 3a (grey dot), showing the difference between the two SAGE composites at $2.2 \mathrm{hPa}$ is mainly caused by the pre-1991 drift in GOZCARDS; this is a result of the conversion of SAGE-II version 6.2 data (used in GOZCARDS) from densities to mixing ratios using NCEP temperatures, while the version 7 SAGE-II dataset (used in SWOOSH) uses MERRA and thereby corrects this issue.

Finally, we show in Fig. 3d the difference between SWOOSH (Tea15) and the latest version (2.6), which sees only minor step changes and short-term variance that appears to line up with instrument changes, except for between 1998 and 2004. Again, it is not clear from this difference plot alone if these changes will lead to a better estimate of ozone variability and trends or not. Further discussion on the SAGE composites is provided in Sect. A2.

\section{Bayesian inference of the underlying ozone time series}

We want to combine the information from the various composites and correctly account for uncertainties, artefacts, and drifts. To this end, we adopt a Bayesian approach to infer constraints on the (unknown) true time series, $\boldsymbol{y}$, given the full set of data, $\mathbf{d}$. The data consist of $n_{c}$ composites, indexed by $c$; each composite is made up of $n_{t}$ measurements, indexed by $t$. A single measurement is hence $d_{t, c}$, where the index ordering is chosen to match that required for the matrix manipulations used in Sect. 3.1. The underlying time series that is to be inferred, $\boldsymbol{y}$, hence has individual elements $y_{t}$.

Bayesian inference necessarily involves conditioning on our knowledge about uncertainties and potential artefacts and drifts, and any prior assumptions about the month-to- 

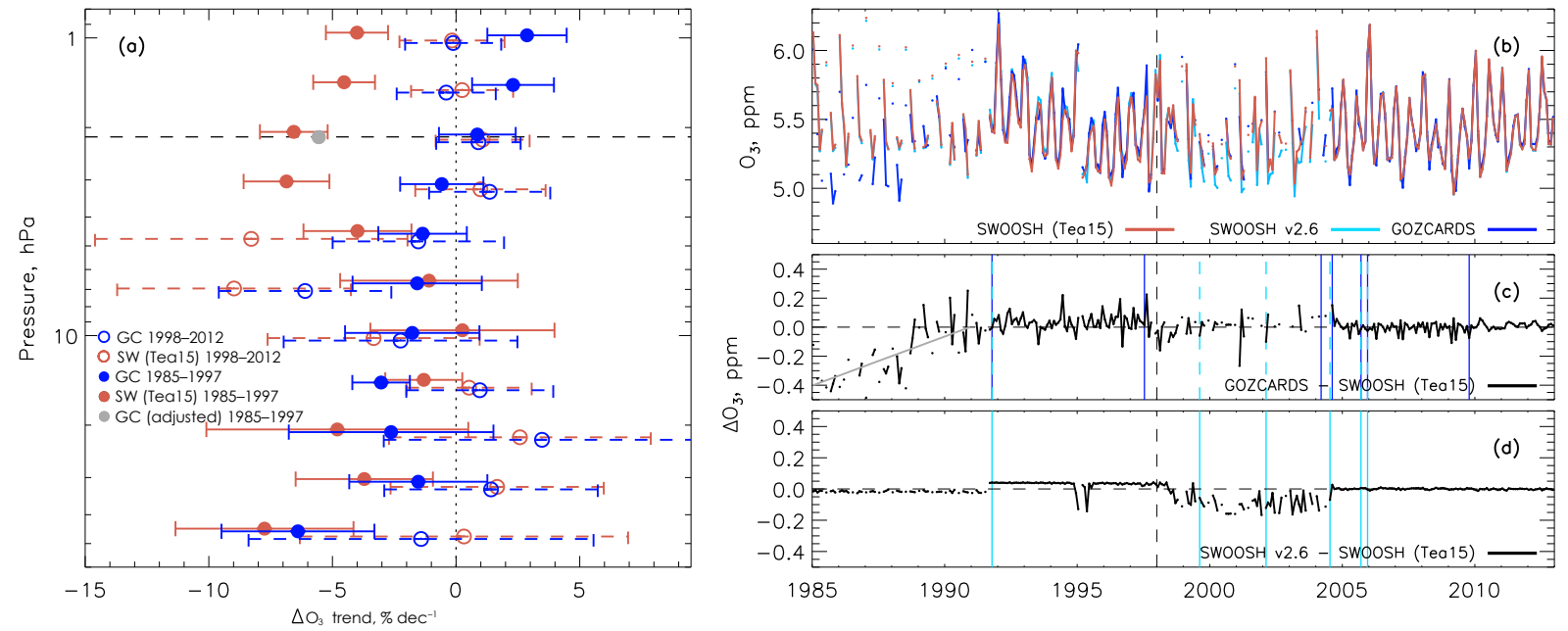

Figure 3. (a) The equatorial $\left(20^{\circ} \mathrm{S}-20^{\circ} \mathrm{N}\right)$ decadal ozone MLR trend profiles for SWOOSH from Tummon et al. (2015) ("Tea15"; red) and GOZCARDS (blue). Dots and solid error bars represent the 1985-1997 trends, and open circles and dashed error bars the 1998-2012 period. A single grey dot is plotted at $2.2 \mathrm{hPa}$, which follows an adjustment to SWOOSH (Tea15) as shown in panel (c) as a grey line. (b) The ozone composite time series for SWOOSH (Tea15) (black), SWOOSH v2.6 (light blue), and GOZCARDS (blue) at $2.2 \mathrm{hPa}$, all shifted to the July 2005-2012 mean of SWOOSH v2.6. (c) The difference between the GOZCARDS and SWOOSH (Tea15) time series is shown in panel (b); the grey line prior to 1991 is an adjustment applied to GOZCARDS to produce the grey dot in panel (a). (d) The difference of SWOOSH-Tea15 and SWOOSH v2.6. The vertical dashed line in panels (b-d) indicates 1 January 1998, which delimits the two periods considered in the MLR results in panel (a). Error bars are $2 \sigma$.

month variability, through our model which we denote as $M$. Bayes's theorem allows us to combine this information in the form of the posterior distribution of the true time series given the data, model, and any prior information $P(\boldsymbol{y} \mid \mathbf{d}, M)$ :

$P(\boldsymbol{y} \mid \mathbf{d}, M)=\frac{P(\boldsymbol{y} \mid M) P(\mathbf{d} \mid \boldsymbol{y})}{P(\mathbf{d} \mid M)}$,

where $P(\boldsymbol{y} \mid M)$ encodes our prior information and assumptions about the month-to-month variability of the underlying true time series, the likelihood $P(\mathbf{d} \mid \boldsymbol{y})$ summarizes our probabilistic model for the data given the associated measurement uncertainties (including our knowledge and assumptions about the possibility of instrumental artefacts systematically biasing the observations at certain times), and the marginal likelihood $P(\mathbf{d} \mid M)$ in this situation just plays the role of a normalizing constant.

In order to form the desired posterior distribution, we require a probabilistic model for the data (Sect. 3.2) that incorporates our knowledge and assumptions about the observational uncertainties (Sect. 3.1), and a clear statement of our prior assumptions (Sect. 3.3). The resulting posterior density is a high-dimensional probability density over $\mathbf{y}$, where the length of the vector $\mathbf{y}$ (i.e. the number of time points in the time series) is typically of order $\sim 10^{2}$. Whilst direct evaluation of such high-dimensional probability densities on a grid is computationally unfeasible, they can be effectively reconstructed through sampling algorithms such as Markov chain Monte Carlo (MCMC), discussed in Sect. 4.

\subsection{Uncertainty estimation}

Our method requires uncertainties for each composite that reflect the actual differences between the reported values and the true state of ozone at the time of each measurement, as encoded in the likelihood (Eq. 7). We cannot use the uncertainties published by the composite teams as they are (in general) not derived in the same way and so they potentially encode information differently. The quoted uncertainties can include (i) uncertainties propagated at each step of the data and composite processing, e.g. in regression analysis used to combine individual instruments; (ii) uncertainties in the absolute offsets; (iii) the total number of observations in each dataset; and (iv) precision and calibration errors. A natural choice might be to scale the uncertainty with the inverse square root of the number of observations used to form the monthly ozone value from each instrument, but this would not correctly deal with systematics such as slow instrument drift (as experienced by the SBUV instruments during the 1995-2000 period). Using the number of data points to weight the monthly mean in each composite would lead to the most likely value simply following the SBUV data almost exclusively until 2005 (see Fig. A2), and drifts would remain in the final product (see Sect. 4.3).

Instead, we seek to estimate the noise level from the data and in particular from the discrepancies between the different composites. Estimating the uncertainties is not the main focus of this paper, so a simple heuristic method is used here, but this is clearly an aspect of this overall data analysis 
problem which should be investigated further. Our approach is based on a principal components analysis (PCA) of the composites to model the differences between them, with the time-dependent noise level of each composite then estimated from the variance of the higher-order components. The starting point of this approach is to treat the full dataset $\mathbf{d}$ as an $n_{t} \times n_{c}$ matrix with elements $d_{t, c}$ as defined above. We then use this to construct the mean-subtracted data matrix $\mathbf{d}^{\prime}$ with elements given by

$d_{t, c}^{\prime}=d_{t, c}-\frac{1}{n_{t}} \sum_{t^{\prime}=1}^{n_{t}} d_{t^{\prime}, c}$,

where each composite is treated separately.

The PCA is implemented via singular value decomposition (SVD) in which the mean-subtracted data matrix is factorized as

$\mathbf{d}^{\prime}=\mathbf{U W} \mathbf{V}^{T}$,

where $\mathbf{U}$ is an $n_{t} \times n_{c}$ matrix in which the columns are the orthogonal component time series, $\mathbf{W}$ is an $n_{c} \times n_{c}$ matrix giving the weights of the components, and $\mathbf{V}$ is an $n_{c} \times n_{c}$ matrix that encodes the contributions of the components to the composites. A standard PCA reconstruction of the (meansubtracted) composites would then have the form

$d_{t, c}^{\prime}=\sum_{c^{\prime}=1}^{n_{c}} U_{t, c^{\prime}} W_{c^{\prime}, c^{\prime}} V_{c^{\prime}, c}$,

where the sum has to go from $c^{\prime}=1$ but is often truncated to include only the first few terms with the highest weights.

Our method of estimating the uncertainties in the composites is based on the above reconstruction formula but is only heuristic in the sense that it does not follow a rigorous calculation. We start by ignoring the leading, i.e. the highest weighted, mode in $\mathbf{U}$ as it is common to all composites, and so it provides no extra information. The various noise artefacts are separated across the other $n_{c}-1$ components, which must be combined somehow to reconstruct the noise. We make the natural choice to weight the modes by their respective contributions to each composite and then sum the resultant variances to obtain uncertainty estimates as

$\sigma_{t, c}^{2}=\sum_{c^{\prime}=2}^{n_{c}}\left(U_{t, c^{\prime}} W_{c^{\prime}, c^{\prime}} V_{c^{\prime}, c}\right)^{2}$.

The steps of this method are illustrated in Fig. 4. The left set of panels shows the SVD applied to ozone at $10 \mathrm{hPa} 0$ $10^{\circ} \mathrm{N}$ : the SVD modes (i.e. from matrix $\mathbf{U}$ ) (black lines; first four panels), each have a different weight (percentage value in the lower right of each plot, from matrix $\mathbf{W}$ ). The first mode contains most of the variance $(84 \%)$ with the remainder split between the other three $(13,1$, and $2 \%)$. The first mode is common to all four datasets, and its relative weight within each dataset is represented by the coloured dots (from matrix $\mathbf{V}$ ) to the right of each mode ranging from -1 to +1 ; the weight of the first mode is similar in all four datasets. The second mode is split roughly equally between the two pairs of composites as indicated by the dots on the right, suggesting that it is the difference between the pairs, and for which the rescaled difference of SBUV-MER and GOZCARDS confirms, plotted in grey and with an almost identical variance to the SVD mode. The SBUV composites have almost zero weight in the third mode, indicating that the mode represents artefacts only within the SAGE composites, again confirmed by the difference between SWOOSH and GOZCARDS (grey). With almost zero weight for the SAGE pair in the fourth mode, the rescaled difference between SBUV pairs confirms the mode represents artefacts in SBUV.

From this, we form the uncertainty estimate for each of the composites in the bottom panel, $\sigma_{t, c}$. Unfortunately, the SVD can only be formed when there are data available in each composite, which leads to gaps, represented by the grey shading in the bottom panel. Because composite sub-periods have different uncertainty characteristics, we fill gaps using the median of the period between instrument changes in the composites (vertical lines in the four modes; colours relate to each composite).

In principle, the time series at each latitude-altitude location in the four composites should be the same, and any deviations from the true value should be a result of one or more of the potential reasons listed in Sect. 4.3. By this assertion, the composites each contain the real time series and an additional set of artefacts. The problem is that we do not know for sure in which dataset a problem might be, especially if the true trend is only apparent in (or missing from) one composite or one composite pair (i.e. SAGE or SBUV based). Thus, the SVD approach allows us to separate the common signal (the leading mode in $\mathbf{U}$ corresponding to the highest weight in $\mathbf{W}$, from those that form the differences between the composites (with lower weights) and the real ozone. This leads to an attribution of higher uncertainty for single datasets that exhibit variance not present in the other three, and allows us to assign higher uncertainties in all the composites when one pair (e.g. SAGE pair) acts differently to the other pair (e.g. SBUV pair). In this way, it is a relatively conservative estimate.

The example at $10 \mathrm{hPa}$ was ideal since modes were easy to associate with artefacts within and between the composite pairs. Another example of the usefulness of applying the SVD approach to estimate the uncertainty is shown for $2.2 \mathrm{hPa}$ and $0-10^{\circ} \mathrm{N}$ in the right-hand panels of Fig. $4 \mathrm{~b}$. The first mode is ubiquitous to the composites, and the fourth mode shows a clear attribution to the SBUV composites (the rescaled difference is shown in grey). However, it is not possible to attribute modes two and three as confidently, though the artefacts are more likely from GOZCARDS and SWOOSH, respectively. Since complete separation of this mode from the other composites is not possible (e.g. that 


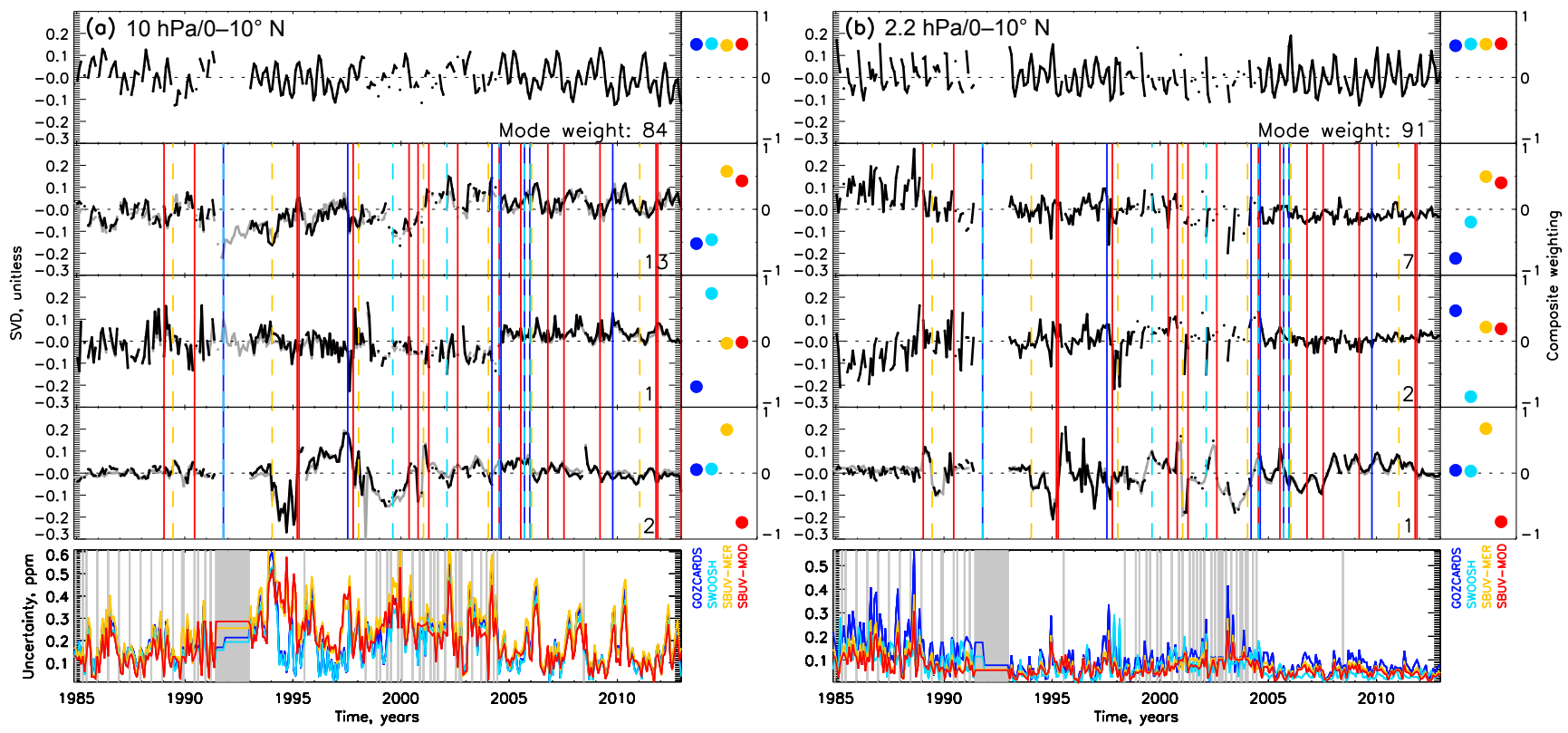

Figure 4. Visualization of the components of the SVD algorithm within the BASIC approach used to estimate the uncertainty on each ozone composite for two examples at (a) $10 \mathrm{hPa}$ and (b) $2.2 \mathrm{hPa}$ at $0-10^{\circ} \mathrm{N}$. The left column of the first four rows show the determined singular value decomposition (SVD) unitless modes (black time series); the mode weighting (\%) is given in the bottom right; the right column is the mode weighting for each composite. All colours represent information related to GOZCARDS (blue), SWOOSH (light blue), SBUV-MER (yellow), and SBUV-MOD (red). Vertical lines represent dates an instrument change in the composite occurred. The grey time series is the arbitrarily rescaled difference between SBUV-MER-GOZCARDS, SWOOSH-GOZCARDS, and SBUV-MER-SBUV-MOD in panel (a) in rows 2-4, and SBUV-MER-SBUV-MOD in panel (b) in row 4. The bottom panel (row 5) in panels (a) and (b) represents the uncertainty derived from the root sum of the squares of the modes (rows) 2-4, weighted by the mode and composite weight, in units of ppm. Grey vertical lines represent dates when data in any composite are missing and filled with the median uncertainty for the sub-period in which they lie (i.e. between the vertical lines in rows 2-4).

SWOOSH is definitely the reason for the third mode), some uncertainty is given to the other composites. This is an intuitive approach to assigning uncertainty to each of the composites.

Satisfyingly, the error estimates display higher uncertainty to individual composites during periods already known to have anomalous behaviour (Sect 4.3). For example, in the lower panel of Fig. 4 at $2.2 \mathrm{hPa}$ (right), GOZCARDS is assigned a particularly high uncertainty during the first 5 years, as expected (Sect. 2.2.2). At $10 \mathrm{hPa}$ (left), the SBUV composites generally have a higher assigned uncertainty, especially around mid-1995, and until 2000, when we know there are instrument drifts in the SBUV composites (Sect. 4.3). In summary, the SVDs allow us to independently and fairly assign an uncertainty to each of the composites.

As the SVD approach is not always able to assign a known artefact explicitly to a specific composite, it is necessary for us to provide additional information regarding the composite uncertainties, whereby in three cases we increase the estimated uncertainty by a factor of 2 . These are (i) when an instrument changes in a composite, which is appropriate since there are many examples of jumps in a composite on, or immediately after, these dates (e.g. Fig. 2c in 1994 and 1995); (ii) during known and significant instrument drifts in SBUV - the SBUV drift from the SVD uncertainty estimate is typically assigned equally to both pairs of composites and so additional information is needed, and tests show that it is only partially accounted for when this additional information is not included - specifically 1995-2000 for both SBUV composites and additionally 1994-1995 in SBUV-MER (these periods are marked by black shading and white text in Fig. 1); and (iii) following the eruption of Mount Pinatubo in SBUVMER only (see Fig. 1 and Sect. 4.3).

\subsection{The likelihood}

With estimates of the uncertainties on each composite, we can construct the joint-likelihood function for the set of composites as a product over the individual likelihoods at each time step (indicated by $t$ ) and composite (indicated by $c$ ), so that

$P(\mathbf{d} \mid \boldsymbol{y})=\prod_{t} \prod_{c} P\left(d_{t, c} \mid y_{t}\right)$,

which implicitly assumes that the measurement errors at different time steps and between different composites are uncorrelated. 


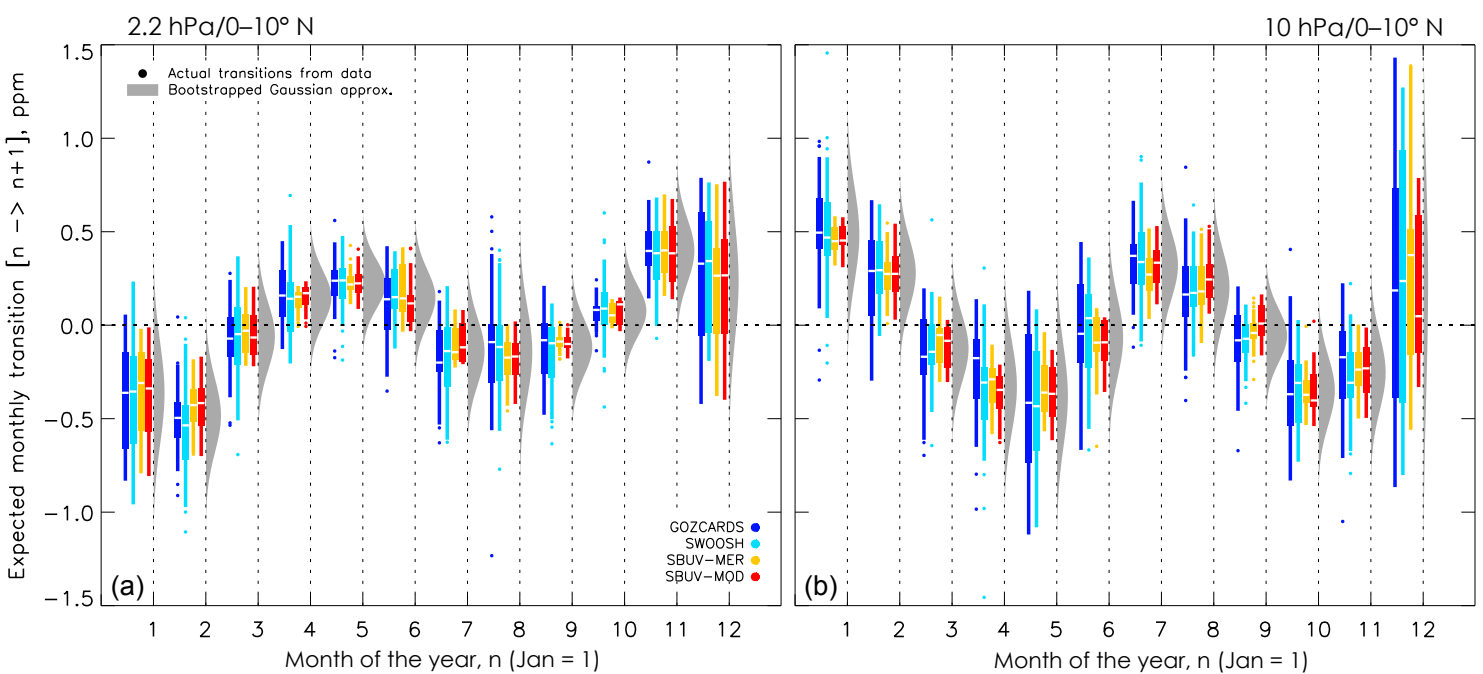

Figure 5. The expected monthly ozone changes (or "transitions") between month $n$ and the next month, $n+1$, i.e. index 1 represents a change between January and February. We show two examples at $0-10^{\circ} \mathrm{N}$ : (a) 2.2 and (b) $10 \mathrm{hPa}$. The box-and-whisker plots are for all observations when no change in the underlying instrument of the composites occurred and represent the interquartile range (IQR) covering the 25 th to 75th percentiles (box) and 1.5 times the IQR or the maximum, whichever is smallest (whisker); outliers are plotted as dots. Plotted to the left of the vertical lines at each index are the changes between months for each composite (represented by the different colours); Gaussian distributions to the right of the vertical lines represent those formed from the mean and standard deviation of all the composite transitions from 1000 bootstraps. These Gaussians are used as transition prior estimates and are calculated for all pressures and latitudes.

A common assumption would be that, ordinarily, the likelihood for a single measurement would be taken to be a normal distribution with a mean given by the true value, $y_{t}$, and a standard deviation of $\sigma_{t, c}$, the measurement uncertainty in composite $c$ at this time step. However, it is clear from even a quick inspection of the data that there are significant disagreements between the different composites, implying several of them - and possibly all - are far more prone to extreme errors (i.e. outliers) than would be predicted by a simple Gaussian likelihood. We hence adopt the model of Box and Tiao (Box and Tiao, 1968) in which there is a probability $0 \leq \beta \leq 1$ that any given measurement has an uncertainty inflated by a factor of $\gamma \geq 1$, such that the likelihood for a single measurement is

$$
\begin{aligned}
P\left(d_{t, c} \mid y_{t}\right) & =\frac{1}{\sqrt{2 \pi} \sigma_{t, c}}\left\{\frac{\beta}{\gamma} \exp \left[-\frac{\left(d_{t, c}-y_{t}\right)^{2}}{2 \gamma^{2} \sigma_{t, c}^{2}}\right]\right. \\
& \left.+(1-\beta) \exp \left[-\frac{\left(d_{t, c}-y_{t}\right)^{2}}{2 \sigma_{t, c}^{2}}\right]\right\} .
\end{aligned}
$$

Smaller values of $\beta$ encode more faith that uncertainties, $\sigma_{t, c}$, are correct; higher values of $\gamma$ correspond to more catastrophic outliers. The standard normal distribution is recovered if either $\beta=0$ or $\gamma=1$. Both $\beta$ and $\gamma$ must either be fixed by hand or kept as hyperparameters to be inferred. We fix $\beta=0.1$ and $\gamma=100$, which implies that we consider outliers reasonably rare but extreme should they occur; this choice leads to multi-modal behaviour as desired (see Sect. A3 and Fig. A3).
When the multiple measurements of the different composites are combined in the product over $c$, the resultant likelihood can be multi-modal when considered as function of $y_{t}$. In cases where the composites disagree, the implication is that it is most likely that one of the measurements is good but not necessarily that which is to be preferred. By contrast, simply multiplying Gaussian likelihoods together in such a situation would result in a joint likelihood that sits between the two (or more) peaks and does not represent likely values according to any of the composites (left column of Fig A3). However, under the model prescribed by Eq. (7), the joint likelihood is multi-modal where subsequent application of the prior may elicit which of the peaks is representative of the truth and which observations were likely dominated by artefacts (or indeed if all composites might be systematically biased simultaneously but in different ways, in which case the resulting posterior for that point will have an inflated uncertainty as desired).

\subsection{Transition prior}

We factorize the prior into a product of transition priors for each month-to-month transition, i.e.

$P(\boldsymbol{y})=P\left(y_{0}\right) \prod_{t=1}^{N-1} P\left(y_{t+1} \mid y_{t}\right)$.

The transition prior provides a way to estimate if measurements of ozone values from the composites in the month being evaluated are more likely or not and hence provide a 
way of assessing anomalous behaviour. The annual, or semiannual, variability that makes up the seasonal cycle, is the largest mode of ozone variability. It is also a relatively consistent mode, so together with information from the observations, it can provide a way to help differentiate between artefacts and real anomalous behaviour.

We form the transition prior from all four composites together. Two examples are given in Fig. 5 at 2.2 and $10 \mathrm{hPa}$ at $0-10^{\circ}$, where the expected change between month $n$ and $n+1$ for the whole year is shown, with, e.g. $n=1$ being the transition between January and February. The monthly changes for all composites are shown with the box-and-whisker plots, which show the mean (white horizontal line), interquartile range (IQR, 25-75th percentiles; thick stem), and full range or 1.5 times the IQR (thin line), with any outliers given as dots; data in a composite where instruments change are not included in the estimates. The grey Gaussian distributions are formed from all the changes between 2 months treated independently and then performing 1000 bootstraps. We note that in the examples shown in Fig. 5, the SAGE-based composites typically have a larger range of month-to-month variance, which we suggest may be due to the higher resolution of the SAGE composite instruments, but we cannot exclude the possibility that this is also related to the low sampling and higher scatter of, e.g. the earlier observations from SAGE-II.

\section{Posterior sampling}

With the likelihood (Sect. 3.2) and prior in hand, we can construct the posterior density for the true time series given the data and our prior knowledge and assumptions, i.e. Eq. (1). The product of the prior (Eq. 8) and the likelihood (Eq. 7) over all the observations gives the numerator of the posterior density defined in Eq. (1). The normalizing denominator cannot be calculated analytically, but fortunately the numerator is sufficient to obtain samples from the posterior distribution. We sample the posterior using Hamiltonian Monte Carlo (HMC) sampling (Neal, 1993) implemented in STAN ${ }^{1}$ (Carpenter et al., 2016); HMC is an MCMC method that is particularly effective at sampling high-dimensional densities (Neal, 1993). The resulting inferred ozone time series forms the BASIC composite.

\subsection{BASIC approach as an approximation to a Bayesian hierarchical state-space model}

When constructing the month-to-month transition prior as described above, we use the data to estimate and fix the prior's hyperparameters, i.e. the means and variances of each month-to-month transition (January-February, FebruaryMarch, etc.). This is using the data twice - once to construct the transition prior and once in the main posterior inference. However, we note that estimating and fixing the hyperparam-

\footnotetext{
${ }^{1}$ STAN software can be found at http://mc-stan.org.
}

eters from the data is an approximation, similar to "empirical Bayes" methods, to a full Bayesian hierarchical treatment where the parameters of the prior would be kept as free unknown parameters and inferred jointly with the true ozone time series. In cases where the hyperparameters are tightly constrained by the data and do not strongly co-vary with the parameters of interest (here the underlying ozone time series), estimating and fixing the hyperparameters from the data before the main analysis is an excellent approximation to the full hierarchical model. ${ }^{2}$

\subsection{Testing BASIC with synthetic data}

We designed synthetic tests to evaluate whether the BASIC approach was effective in retrieving the "true" ozone time series given a set of four ozone composites that had jumps, drifts, and noise, similar to those we encounter in the existing datasets. Overall, we found the BASIC approach to be successful at estimating ozone and, in particular, better than any individual composite that contains artefacts. These synthetic tests are presented in Sect. A5.1.

The BASIC composite result for the $0-10^{\circ} \mathrm{N} 2.2 \mathrm{hPa}$ time series is given in Fig. 6a, with all four composites, and the BASIC composite with uncertainties at 2 standard deviations (dotted lines) and 68, 95, and 99\% credible intervals (dark, medium, and light grey shading); the differences in Fig. 6a relative to the BASIC composite are shown in Fig. 6b. It is clear that the BASIC approach has successfully accounted for (i) the early drift prior to 1991 in GOZCARDS resulting from the use of NCEP reanalysis temperatures and (ii) the

\footnotetext{
${ }^{2}$ We leave a more careful hierarchical analysis to future work, expecting this approximation to have a small impact on the results, but outline the full hierarchical model briefly below for completeness. In the generative hierarchical model, the true ozone time series are generated from the transition prior as

$\begin{aligned} y_{t} & =y_{t-1}+\Delta_{t} \\ \Delta_{t} & \sim \mathcal{N}\left[\mu_{m(t)}, \sigma_{m(t)}\right],\end{aligned}$

where the mean $\mu_{m}$ and variance $\sigma_{m}$ depend only on the month of the year, $m(t)$, corresponding to the time step $t$, and broadly capture the stochastic month-to-month variability as described above. The individual composite datasets are then generated from the Gaussianmixture model described in Eq. (7) as

$d_{t, c} \sim(1-\beta) \mathcal{N}\left(y_{t, c}, \sigma_{t, c}^{2}\right)+\beta \mathcal{N}\left(y_{t, c}, \gamma^{2} \sigma_{t, c}^{2}\right)$,

where $\beta$ and $\gamma$ describe the outlier rate and outlier uncertainty inflation factor, respectively, and $\sigma_{t, c}$ is the assumed measurement uncertainty. Since in general we do not know the hyperparameters of the prior $\left(\mu_{m}\right.$ and $\left.\sigma_{m}\right)$ or the Gaussian-mixture nuisance parameters $(\beta$ and $\gamma)$ a priori, the most principled Bayesian solution is to infer the joint posterior distribution for the true ozone time series $\mathbf{y}$ and the hyper and nuisance parameters together, and formally marginalize over the latter. We leave this full treatment to future work and here estimate and fix the prior hyperparameters, and choose the Gaussian-mixture parameters heuristically.
} 
high scatter in both the SAGE composites prior to 1991 and mainly in SWOOSH prior to 2004 resulting from the low sampling of the occultation instruments used. When disagreement between composites increases, or the priors inflate the uncertainties, the BASIC composite uncertainty estimate naturally inflates to allow for the higher uncertainty during that period; on the other hand, the BASIC composite uncertainties reject most of GOZCARDS prior to 1989 by being outside the $99 \%$ credible interval.

Another example, at the higher pressure of $10 \mathrm{hPa}$, is given in Fig. $6 \mathrm{c}$ and d. Here, we see that the BASIC approach has accounted for (i) the SBUV-MER problem following the Mt. Pinatubo eruption, during which SBUV-MOD measurements are not provided, (ii) rapid steps in the SBUV composites between 1995 and 2001, and (iii) some of the drifts in the SBUV composites during the same period. What is clear here, especially in the period after 2002 , is that while the BASIC composite reproduces most of the variance, it cannot determine whether the higher amplitude variance of the QBO signal in the SAGE composites is more likely to be correct than the SBUV composites, though we know the reason is due to the lower vertical resolution of the SBUV-type instruments and that the QBO represented by the SAGE composites is more likely to be correct (see Sect. 4.3). We do not currently have a solution for this particular issue, though the errors do inflate naturally to accommodate this uncertainty, and so typically within the uncertainties this issue is captured by the BASIC approach.

Finally, to show how the BASIC approach operates in a completely different regime to that near the Equator, in Fig. 6e and $\mathrm{f}$ we give an example at $6.8 \mathrm{hPa}$ and $50-40^{\circ} \mathrm{S}$. Here, ozone lacks a semi-annual component of variability. Except for between 1993 and 2001, all four composites show broadly similar variability. The SAGE composites again appear to show spikes that are not present in the SBUV composites, and indeed on many occasions do not occur in both SAGE composites. Therefore, many of these are rejected by the BASIC composite. We cannot discount that some of these artefacts are a result of the better resolution in the SAGE composites and may be real, for example, unexplained artefacts after 2008, but these are generally found to remain at or within the $99 \%$ credible interval. Following the instrument change in SBUV-MER in 1994, and until 2001, we see anomalous behaviour in SBUV-MER that is rejected by the BASIC composite at the $99 \%$ level throughout this period; between 1995 and 1997, SBUV-MOD also displays behaviour quite different to the other composites, and this is also generally rejected.

\subsection{Further examples of problems resolved by the BASIC approach}

In Sect. 2.2.1 and 2.2.2, we showed examples of differences between composites based upon the same, or similar, instrument data. It is not always clear by looking at the pairs of composites, however, which is more likely to be correct: drifts and rapid changes occurring over a few months cannot be immediately attributed to a specific composite. However, as we will now demonstrate, additional information from the literature, knowledge of when instruments are added or removed within the composites, and looking at the differences of all four composites at the same time, helps to build confidence in attributing the source and reason for the deviation, and then correcting it - these are encoded in the uncertainties of each composite as discussed in Sect. 3.1. We also show the effectiveness of the BASIC approach in accounting for most of these artefacts. The final BASIC ozone composite product that integrates information from all four composites is denoted "BASIC". However, it is also possible to only use information from either the SBUV pair ("BASIC(SBUV)") or SAGE pair ("BASIC(SAGE)") of composites (with SVD uncertainties and transition priors constructed using only the respective pairs of data), which elucidates how the prior information applied in the BASIC algorithm is able to perform if information is missing from the other pair of composites. In other words, a correction of artefacts (e.g. drifts in SBUV composites) that do not appear in differences of just one composite pair strengthens our claim that the BASIC approach is correctly accounting for artefacts in the composites. For clarity in the figures introduced here, we do not provide uncertainties on the BASIC results presented.

In Fig. 7, we show two examples of the four ozone composites at $0-10^{\circ} \mathrm{N}$, at $2.2 \mathrm{hPa}$ (Fig. $7 \mathrm{a}-\mathrm{g}$ ) and $4.6 \mathrm{hPa}$ (Fig. 7h-n). Below the absolute time series (Fig. 7a and $h$ ) are six plots (Fig. 7b-g and $i-n$ ), which are the differences between each pairing of composites (black); the absolute BASIC composite ozone is shown with a dotted line, and differences of the BASIC, BASIC(SAGE), and BASIC(SBUV) compared to the composites are given in red, blue, and orange, respectively. Once again, the early drift (e.g. Fig. 7b SWOOSH - GOZCARDS) and the steps (e.g. Fig. 7n SBUV-MER-SBUV-MOD) are clearer in these restricted latitude bands than in the broader equatorial band presented in Figs. 2c and 3c. However, considering these different pressures and latitudes, and the SBUV-SAGE differences (Fig. 7c-f), additional anomalous behaviour is revealed, which we list and discuss in the following.

1. The most significant problem in creating a unified calibration for all SBUV instruments is the orbital drift (McPeters et al., 2013). Ideally, the local time at Equator crossings should be the same each orbit, and the orbit should be near polar to attain near-global coverage. However, NOAA satellites slowly drifted over time, changing from near 14:00 LT (local time) (10:00 LT, NOAA-17) Equator crossings to late afternoon (early morning, NOAA-17) Equator crossings. NOAA-9, -11, -14 , and -16 drifted through the terminator and began making early morning measurements. The Equatorcrossing time for each of the SBUV satellites is shown 

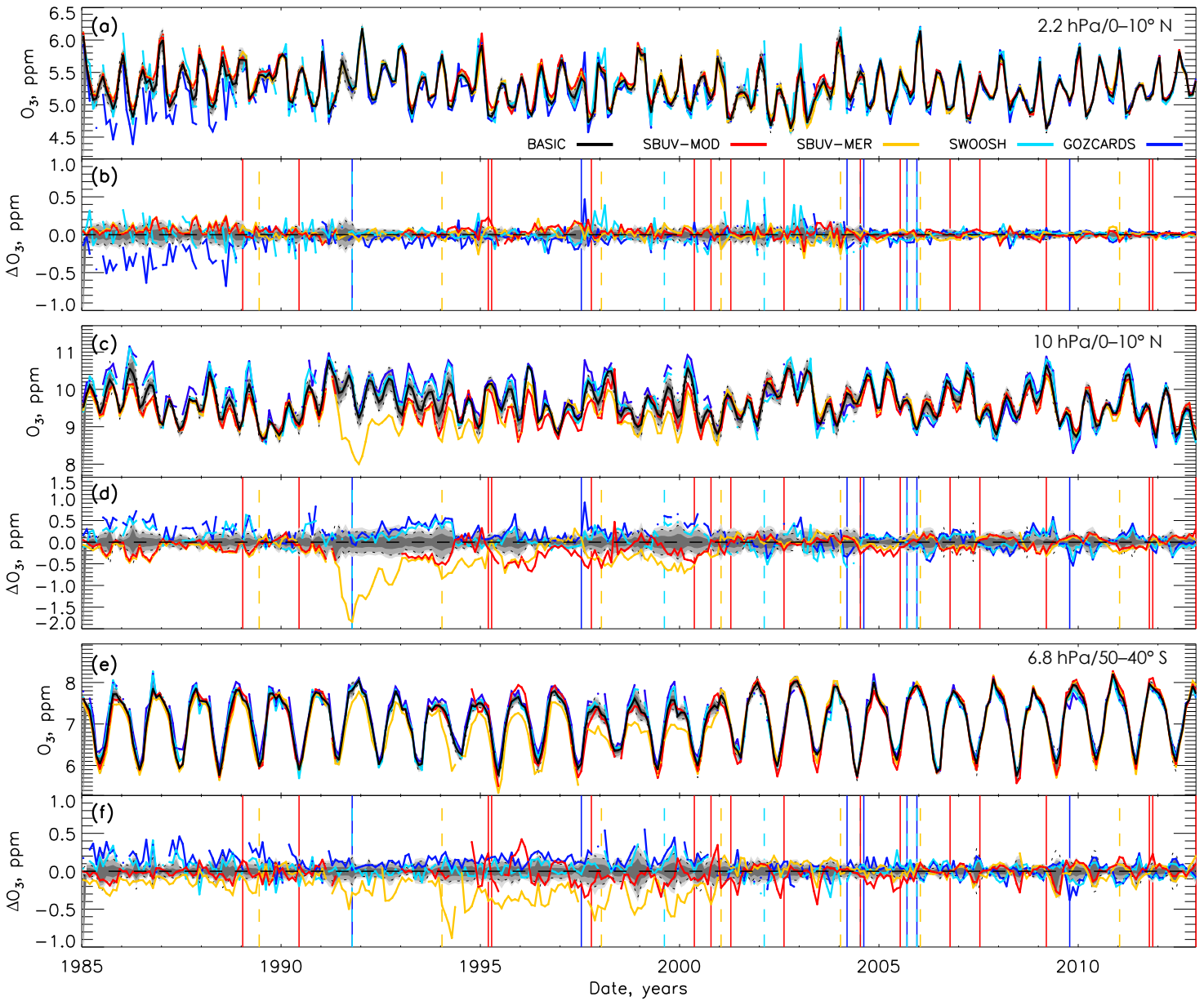

Figure 6. Ozone time series at three stratospheric locations from 1985 to 2012, all bias shifted to the mean of SWOOSH after August 2005. (a) Absolute ozone at $2.2 \mathrm{hPa}$ over $0-10^{\circ} \mathrm{N}$ from SWOOSH (light blue), GOZCARDS (blue), SBUV-MER (yellow), and SBUV-MOD (red). The BASIC composite mean estimation (black) is plotted with shading representing $68 \%$ (dark grey), $95 \%$ (grey), and $99 \%$ (light grey) credible intervals (CIs); these CIs are not Gaussian, so 2 times the standard deviation is also plotted with thin dotted lines. Panel (b) is the same as (a), but now for the difference relative to the BASIC composite. Panels (c) and (d) are as the same as (a) and (b) at $10 \mathrm{hPa}$ and $0-10^{\circ} \mathrm{N}$, and (e) and (f) are the same as (a) and (b) at $6.8 \mathrm{hPa}$ and $50-40^{\circ} \mathrm{S}$. Vertical dashed and solid lines in panels (b), (d), and (f) identify changes in the instruments used in the composites.

in Fig. 1 between the SBUV-MOD and -MER composite information. Any instrument or calibration errors may be significantly enhanced for observations taken as the orbit approaches the terminator, such that the orbit drift can lead to an apparent time-dependent trend in ozone that could be misinterpreted as real; McPeters et al. (2013), DeLand et al. (2012), and Bhartia et al. (2013) do not recommend the use of near-terminator data for this reason. Accordingly, SBUV-MOD, with the exception of NOAA-11, does not include any observations taken outside the 08:00-16:00 LT equatorial crossing time range (marked as dotted horizontal lines in Fig. 1) and similarly SBUV-MER prioritizes measurements made while instruments are in their optimum orbits. The clearest example of this drift-related trend can be seen in Fig. 7k, m, and $\mathrm{n}$ in all differences with respect to SBUV-MOD between 1995 and 1998 (until 2000 with respect to SBUV-MER in Fig. 7n); there is then a reversed drift until after 2000. The differences with the SAGE composites indicate that a 1994-1995 drift is likely in SBUV-MER from the exclusive use of NOAA-9; for 1995-1997, the drift is probably in both but more prominent in SBUV-MER differences; the 1997-2000/2001 drift is more likely in SBUVMER with the exclusive use of NOAA-11 (SBUV-MOD merges NOAA-11 with NOAA-14). Other smaller drifts between the SBUV composites are visible in Fig. 7, e.g. in 2001 and 2002. While BASIC(SBUV) and BASIC were able to account for the large discontinuity present in Fig. 7n, BASIC(SBUV) is unable to account for the 1997-2000 drift in SBUV-MOD. We do inform the BASIC approach that the uncertainties should be increased 

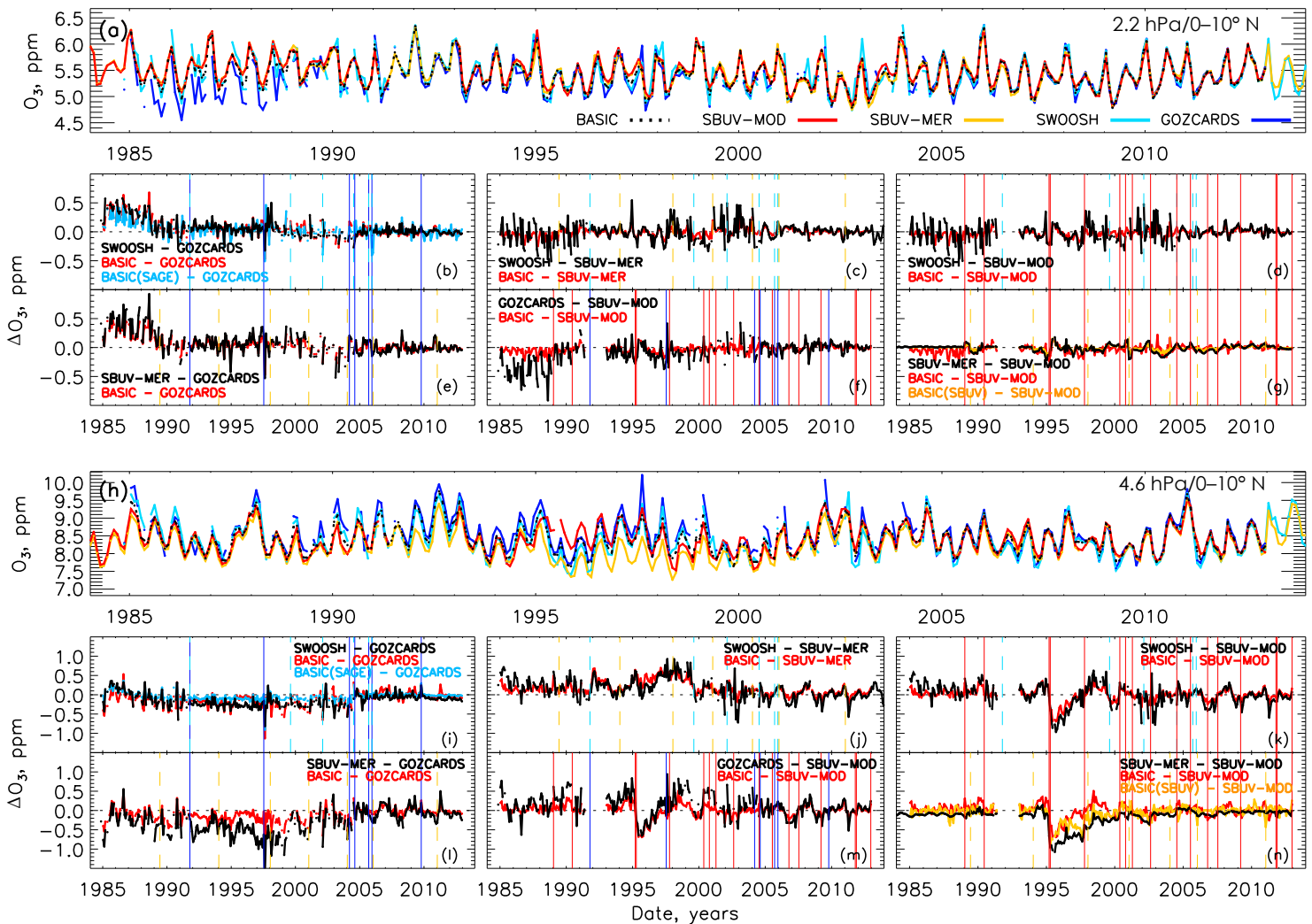

Figure 7. Ozone time series at two stratospheric locations from 1984 to 2014, all bias shifted to the mean of SWOOSH after June 2005. (a) Absolute ozone at $2.2 \mathrm{hPa}$ over $0-10^{\circ} \mathrm{N}$ from SWOOSH (light blue), GOZCARDS (blue), SBUV-MER (yellow), and SBUV-MOD (red). (b-g) The difference between each pairing of the four composites and with the BASIC composite (see legends). Panels (h-n) are the same as $(\mathbf{a}-\mathbf{g})$ but at $4.6 \mathrm{hPa}$ and $0-10^{\circ} \mathrm{N}$. Solid and dashed vertical lines represent months with a change in the instrument used to construct the composite (colours are with respect to the composite colour in panels $\mathbf{a}$ and $\mathbf{h}$ ).

in the SBUV composites during the drift period 19952000 (from 1994 in SBUV-MER), so uncertainties are equal for this period in BASIC(SBUV). Nevertheless, with the inclusion of the SAGE composites this drift can be accounted for (red line in Fig. 7j-n), which further reinforces the need for information from all composites to resolve problems. Confirmation of drift problems during the periods mentioned (DeLand et al., 2012; McPeters et al., 2013; Kramarova et al., 2013b; Frith et al., 2014) justifies using it as prior information to down-weight these data for this time (see Sect. A1 for more information).

2. The apparent high scatter at $2.2 \mathrm{hPa}$ in all differences involving SAGE composites (i.e. Fig. 7b-f) during the periods of 1985-1991 and 1997-2004 coincides with periods when only occultation instruments were active (SAGE-II, UARS/HALOE, and ACE-FTS). Toohey et al. (2013) and Sofieva et al. (2014) convincingly demonstrated that insufficient and/or inhomogeneous sampling can result in inaccurate monthly estimates and even induce spurious spikes in ozone time series; coarse-sampling occultation-type instruments such as GOMOS and ACE-FTS can lead to differences of up to $20 \%$. This can especially affect seasonal cycle representation, especially at high altitudes where ozone undergoes rapid variations with latitude and time of day. This is why spurious variability from occultation instruments is clearly evident in Fig. 7 during the aforementioned periods. Even though satellite measurements from limb viewers have a lower vertical resolution than occultation, these are still sufficient to reduce the monthly zonal-mean scatter in the SAGE-based composites when overlaps with occultation instruments occur (e.g. 1992-1997 in GOZCARDS). The BASICGOZCARDS difference in Fig. 7e agrees closely with the month-to-month artefacts that are highlighted in the SBUV-MER-GOZCARDS difference. This is not because of the information provided in the SBUV composites, which do not display this behaviour, but because the deviation from the natural seasonal cycle is so high that the month-to-month seasonal variability is more informative. This is confirmed by the high agreement between BASIC-GOZCARDS with BASIC(SAGE)- 
GOZCARDS on these short timescales in Fig. 7b, the latter of which contains no knowledge from the SBUV composites.

3. The drift between the SAGE composites prior to 1991 (Fig. 7b and i; see Sect. 2.2.2) is largely absent in the SWOOSH composite compared to SBUV composites (Fig. 7c and d), confirming it as a feature of GOZCARDS only. It is clear from Fig. 7e that the artificial trend in GOZCARDS prior to 1991 is fully accounted for by BASIC, and once again the agreement of BASIC-GOZCARDS with BASIC(SAGE)GOZCARDS in Fig. 7b shows that the information in the SAGE composites alone is sufficient to eliminate most, though not all, of this problem. No prior information about the drift being in GOZCARDS is provided to the BASIC approach - the ability for the BASIC approach to account for the drift is most likely because SWOOSH agrees with the prior information from the seasonal variability (in the transition prior) much better than GOZCARDS.

4. A small downward step in the SAGE composite difference in Fig. 7b and i in 2004 occurs around the time both SAGE composites have an instrument change. This feature is more evident in the differences between GOZCARDS and the SBUV composites than for SWOOSH, at both altitudes. At the lower altitude of $4.6 \mathrm{hPa}$ in Fig. 7i, it appears that BASIC(SAGE) could not account for the jump in GOZCARDS and ends up slightly offset from the black difference line. The BASIC approach performs better with the additional information provided by the SBUV composites and fully accounts for this jump.

5. A prominent feature in Fig. $7 \mathrm{j}-\mathrm{m}$ is the approximately 2- to 3-year oscillation. This is the result of lower vertical resolution in the SBUV observations, which leads to a damping of the quasi-biennial oscillation (QBO) signal in SBUV relative to the higher resolution instruments of the SAGE-based composites; at $3 \mathrm{hPa}$ SBUV has a vertical resolution of approximately $6-7 \mathrm{~km}$, while the SAGE-based instruments are usually better than $3.5 \mathrm{~km}$ - the vertical resolution only gets larger for SBUV with lower altitude, reaching a maximum of $\sim 15 \mathrm{~km}$ below the tropopause (Bhartia et al., 2013). After 2003, the resolution effect is more clearly visible in Fig. 7h, since many of the other instrumentdata/composite artefacts are absent. Kramarova et al. (2013a) showed that by applying the SBUV resolution kernel to higher vertical-resolution Aura/MLS data led to good agreement with SBUV data. Focusing on the period after 2005 in Fig. 7h-n, it is evident that BASIC is unable to distinguish between the QBO represented in the SAGE and SBUV composites; this is because uncertainties are similar during this period and composite issues are generally absent. We discuss this further in Sect. A5.2.

6. Following the eruption of Mt. Pinatubo in June 1991, there is a large drop in SBUV-MER at 10 and $16 \mathrm{hPa}$ due to interference in viewing from volcanic aerosols (not shown here, but see Fig. 6c and d), which is absent in the SAGE composites; SBUV-MOD does not include data during this period. Ozone is usually depleted by sulfate aerosols following a volcanic eruption but at lower altitudes. Due to the rapid departure of SBUV-MER from the SAGE composites, the BASIC composite predicts that the SAGE composites are more likely to be correct during this period. To be clear, the BASIC approach can adapt to rapid, unexpected changes in ozone: if all the datasets had shown a sudden and similarly large change that was significantly different from the prior expectation for that month, it would tend towards a tighter cluster of observations as more likely than the broader prior estimate. We discuss this period further in Sect. A5.2.

7. For completeness, steps in the SBUV composites in Fig. 7k, m, and n, discussed in Sect. 2.2.1, occur in 1995 and in 2003, 2004, and 2007 in Fig. 7n; though these are not the only times that steps occur; prominence of steps depends on altitude and latitude. The BASIC approach accounts for these discontinuities, which is most clear for the large jump in the SBUV-MOD composite in Fig. 7k, m, and n; absence of a jump in Fig. 7i confirms the success of the BASIC approach. For the BASIC(SBUV)-SBUV-MOD case in Fig. 7n (orange), which relies exclusively on the SBUV composites, the large step in 1994/1995, and drift that follows, is mostly accounted for.

\section{Results}

Now that we have established the validity of the BASIC approach and constructed an ozone composite from GOZCARDS, SWOOSH, SBUV-MOD, and SBUV-MER, we turn to analysing trends and modes of variability. This is often performed using MLR (WMO/UNEP, 1994; Soukharev and Hood, 2006; Chiodo et al., 2014; Kuchar et al., 2015; Harris et al., 2015). However, the use of DLM, first applied to ozone data by Laine et al. (2014), appears to be more robust at estimating the background trend, especially if it is nonlinear. Laine et al. (2014) noted this when comparing their DLM results with the MLR results of Kyrölä et al. (2013) where linear trends were sometimes found to be inverse to those estimated using DLM. We performed tests upon the artificial time series used to evaluate the performance of both methods with the BASIC approach (Sects. 4.2 and A5.1). We briefly introduce both methods below. We compare their performance on the artificial time series and the BASIC correction, introduced in Sect. A5.1, in Sect. A6. We found that in 
every test case the DLM did equally well, or better, at estimating the true background "trend" than the linear estimate from MLR (see Figs. A9 and A10) both for non-linear background trends and for time series with large artefacts.

\subsection{MLR analysis}

We perform MLR analysis on deseasonalized time series (i.e. by subtracting monthly means) using five regressors: the F30 radio flux (solar), which is superior to the $\mathrm{F} 10.7 \mathrm{~cm}$ radio flux for representing solar UV variability (Dudok de Wit et al., 2014); the stratospheric aerosol optical depth (SAOD; Sato et al., 1993, for volcanic eruptions); the El Niño-Southern Oscillation (ENSO); and two orthogonal modes of the dynamical quasi-biennial oscillation (QBO). These regressors are displayed in the upper part of Fig. 1. When we analyse decadal trends between 1985-1997 and 1998-2012, we use a linear trend to estimate the long-term trend. We use prewhitening and a first-order autoregressive process (AR1) to account for autocorrelation in the residuals (Tiao et al., 1990). Statistical significance of the regression coefficients was evaluated with a Student's $t$ test.

\subsection{DLM analysis}

We perform a DLM analysis following very closely the model and formalism of Laine et al. (2014). We use the same five regression components as in the MLR. We allow for two modes of seasonal variability in the fit (with 6- and 12-month periods), where additional (Gaussian-process) variability of the sinusoidal seasonal modes is also allowed for (following Laine et al., 2014), and variance of the (Gaussian) seasonal model variability $\sigma_{\text {seas }}^{2}$ is kept as a free parameter in the fit. We include an AR1 process, where the variance $\sigma_{\mathrm{AR}}^{2}$ and correlation coefficient $\rho_{\mathrm{AR}}$ of the AR process are also kept as free parameters in the fitting process. In contrast to MLR, the DLM approach allows for a fully non-linear "trend", where the degree of non-linearity $\sigma_{\text {trend }}$ is also kept as a free parameter in the fit (see Laine et al., 2014 for details). In further contrast to MLR, the Bayesian DLM approach jointly fits for the non-linear time-varying trend, the regression coefficients of the five proxies and seasonal modes, as well as the nuisance parameters $\sigma_{\text {seas }}, \sigma_{\mathrm{AR}}, \rho_{\mathrm{AR}}$, and $\sigma_{\text {trend }}$; uncertainties in the nuisance parameters and regression coefficients are formally marginalized over when stating inference of the trend, leading to a principled propagation of uncertainties. Similarly, uncertainties in the nuisance parameters and trend can be marginalized over when we are interested in the regression coefficients.

Our DLM analysis follows Laine et al. (2014) except for some small differences in the prior choices. For $\sigma_{\text {trend }}$, we use a positive half-Gaussian prior with zero mean and dispersion 0.0005 . For $\sigma_{\text {seas }}$ and $\sigma_{\mathrm{AR}}$, we take positive uniform priors over $[0, \infty]$, and for the correlation coefficient of the AR process we take a uniform prior over $[0,1]$, assuming that negative correlations are unphysical in this context. We also do not impose an external prior on the initial value of the AR process, as is done in Laine et al. (2014), but draw the initial value of the AR process from its stationary distribution, i.e. $\mathcal{N}\left(0, \sigma_{\mathrm{AR}} / \sqrt{\left(1-\rho_{\mathrm{AR}}^{2}\right)}\right)$. Recovery of the DLM parameters $\left\{\sigma_{\text {trend }}, \sigma_{\text {seas }}, \sigma_{\mathrm{AR}}, \rho_{\mathrm{AR}}\right\}$ under the chosen priors is shown in a set of figures in Sect. A4. As in Laine et al. (2014), we use MCMC to sample the joint posterior of the DLM parameters, regression coefficients of the proxies, seasonal cycle, and non-linear trend.

\subsection{Multi-decadal changes in ozone}

Here, we present estimates of changes in ozone between 1985 and 1997, and between 1998 and 2012 (Fig. 8). This is the first time that DLM has been applied to these composite datasets, including recently updated SWOOSH and SBUVMER. While we focus on the DLM results, we also refer to results using MLR given in Fig. A11.

Typically, ozone trends are reported as linear decadal percentage changes in three latitude bands in the Southern Hemisphere $\left(60-35^{\circ} \mathrm{S}\right)$, over the Equator $\left(20^{\circ} \mathrm{N}-20^{\circ} \mathrm{S}\right)$, and in the Northern Hemisphere $\left(35-60^{\circ} \mathrm{N}\right)$ with sub-periods ending and starting in December 1997 and January 1998, respectively, as shown in Fig. 8 (Fig. A11 for MLR) (WMO, 2014; Tummon et al., 2015; Harris et al., 2015). These integrated latitude bands were formed by averaging the area/latitude-weighted $10^{\circ}$, with the $30-40^{\circ}$ band receiving half the weight of the equivalent full band; the resultant time series were then analysed.

It does not make sense to provide a linear trend estimate for the non-linear DLM background trend. Instead, in Fig. 8 we give the percentage change of ozone between the first and last months of the sub-periods, i.e. between January 1985 and December 1997 (top row), and January 1998 and December 2012 (lower panels). Uncertainties represent the $95 \%$ credible intervals of the change for all 100000 samples estimated with the DLM algorithm (shading for BASIC, bars for all others). Since we do not show decadal trends for the DLM (but do for MLR in the Appendix), we also show as dashed black lines in Fig. 8 the mean MLR-BASIC linear trend profiles from Fig. A11, scaled from decadal changes to the longer 13- and 15-year sub-periods.

In the earlier period (1985-1997), the DLM and MLR profiles agree well (within the DLM uncertainty). The DLMBASIC typically displays better agreement with the GOZCARDS profiles than the others in the northern and southern midlatitudes, but the mean profile is generally closer to that of SBUV-MOD over the Equator. Indeed, above $4 \mathrm{hPa}$, SWOOSH is typically at or outside the BASIC composite $95 \%$ credible interval in northern and equatorial bands (this is also the case with MLR). Interestingly, the SBUV composites are often outside the MLR-BASIC uncertainty range above $7 \mathrm{hPa}$ at midlatitudes in both hemispheres; DLM un- 

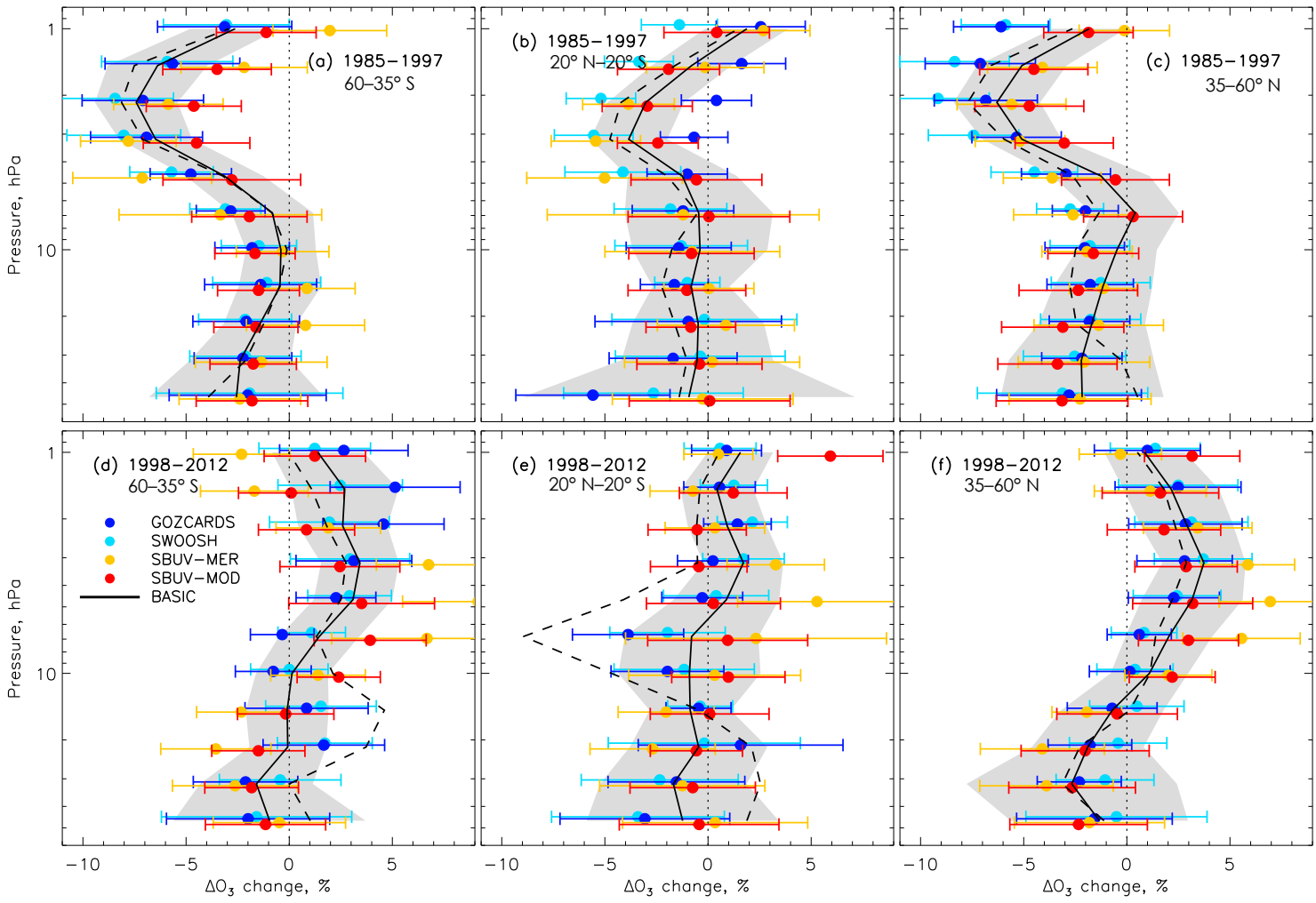

Figure 8. The percentage change in ozone from DLM between 1985 and 1997 (a-c), and 1998 and 2012 (d-f), over 60-35 ${ }^{\circ}$ S (a, d), 20 $0^{\circ}$ $20^{\circ} \mathrm{N}$, and $35-60^{\circ} \mathrm{N}(\mathbf{c}, \mathbf{f})$. GOZCARDS, SWOOSH, SBUV-MER, and SBUV-MOD are shown with error bars representing $95 \%$ credible intervals; for the BASIC composite (black), shading represents uncertainties. The mean linear trend estimate from MLR for the BASIC composite is given as a black dashed line (no uncertainties) and is the scaled version of the MLR-BASIC decadal trend shown in Fig. A11.

certainties are larger and the four composites are in closer agreement when trends are analysed using DLM. This might hint that MLR is being biased by residual variance and/or underestimating error bars, in contrast to DLM, as was observed in the test cases (see Sect. A6). Overall, the 1985-1997 DLM results are consistent with previous studies and MLR, with a significant decline in ozone above $7 \mathrm{hPa}$ at all latitudes, especially at midlatitudes, and negative but usually insignificant trend at lower altitudes.

The results for the latter period, 1998-2012, show a significant positive trend in the upper stratosphere above $7 \mathrm{hPa}$, as expected to occur following the implementation of the Montreal Protocol. The result is significant in every dataset analysed with DLM in both the northern and southern midlatitudes for at least one pressure level; for the BASIC composite, the result is clear at multiple altitudes. We note that the MLR results are only statistically significant at northern midlatitudes for both SBUV composites and for all composites in the southern midlatitudes at 3.2 and $4.6 \mathrm{hPa}$. There are also statistically significant differences between the mean MLR-BASIC and the DLM-BASIC profiles over the Equator and at northern midlatitudes; in the southern region, DLM profiles for composites are less consistent than when using MLR, but the DLM-BASIC results are in good agreement.
The DLM profile shapes in the Northern Hemisphere are consistent with each other, with a negative trend in the lower stratosphere, though usually insignificant at the $95 \%$ level, and a positive response in the upper stratosphere, confirming the result of Harris et al. (2015). Interestingly, with the exception of SBUV-MOD, the large and significant negative MLR equatorial trends seen in most of composites at $7 \mathrm{hPa}$ disappear when using DLM, except in GOZCARDS. This anomaly was found in an integrated set of seven composites by Harris et al. (2015), though not in the multi-model mean of the same composites in Tummon et al. (2015). These results suggest that it may be an artefact of the analysis approach rather than a real feature and further investigation is required.

In Fig. 9, we plot the DLM moving trends as a percentage change in ozone relative to 1998; only the BASIC composite uncertainty is presented ${ }^{3}$, and the MLR-BASIC linear trends pre-1998 and post-1997 are given as dashed lines; as a guide the MLR uncertainties are typically smaller than the DLM (see Fig. A11). From Fig. 9, significant disagreement at 5-

\footnotetext{
${ }^{3}$ The uncertainties presented in Fig. 9 include an uncertainty on the absolute level in addition to that of the trend, while those presented in Fig. 8 contain only the uncertainty in the change.
} 
(a) $60-35^{\circ} \mathrm{S}$

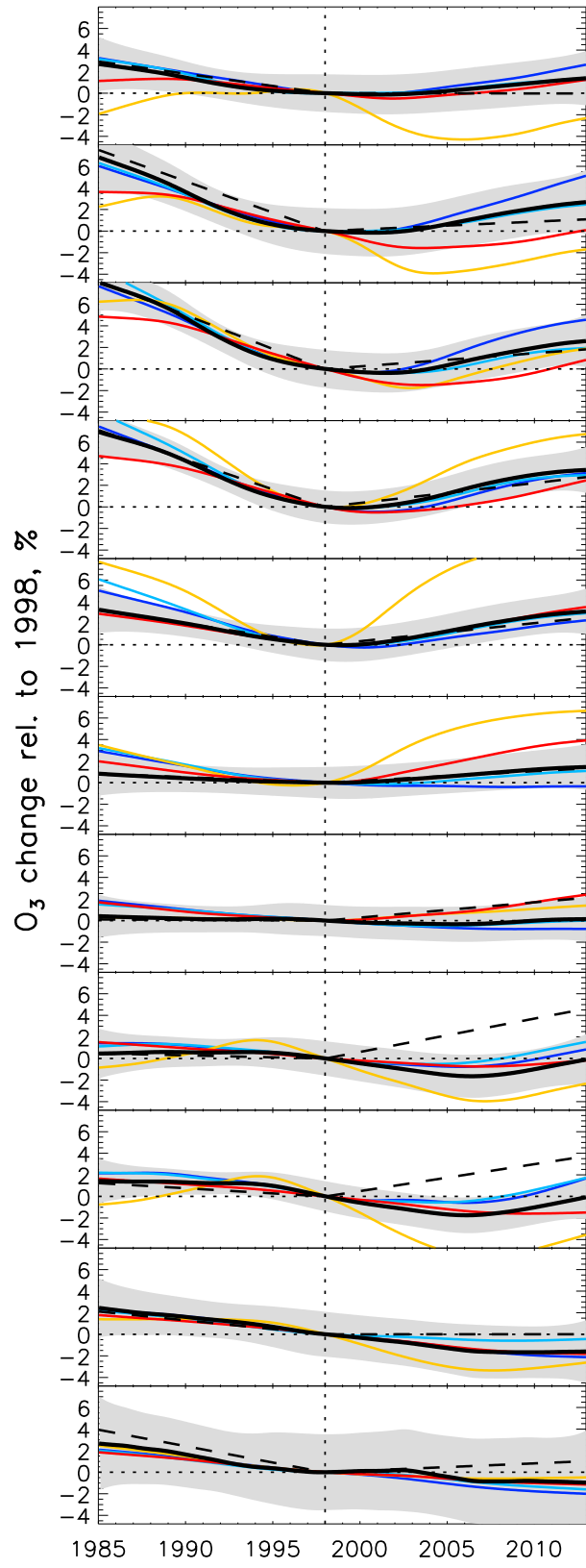

(b) $20^{\circ} \mathrm{S}-20^{\circ} \mathrm{N}$

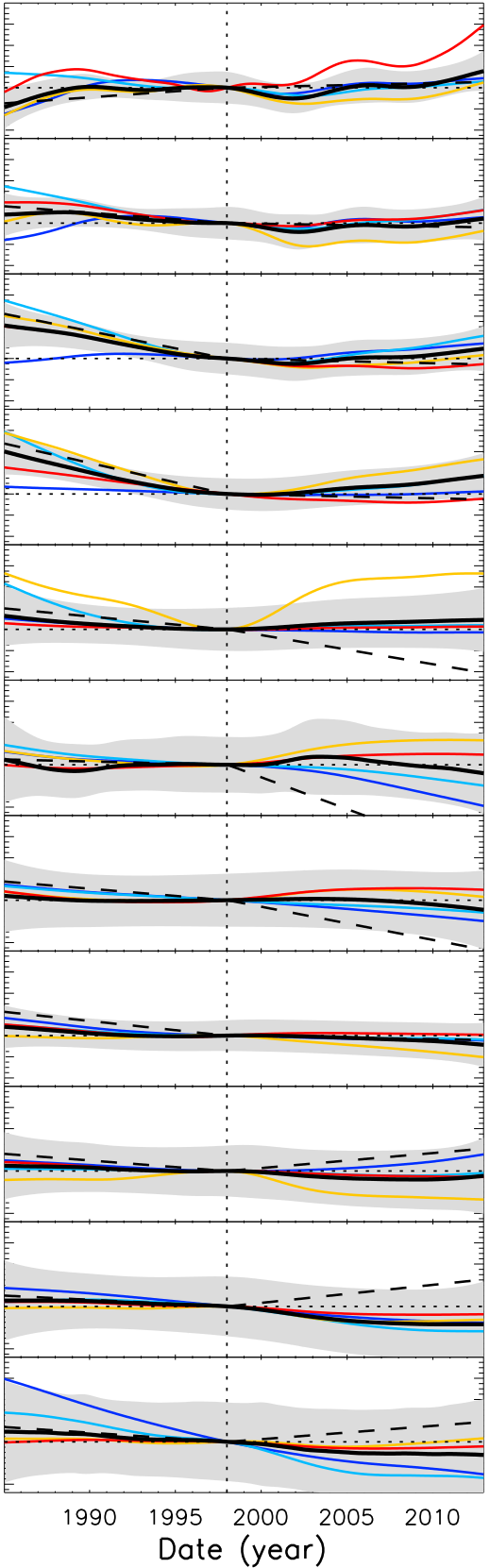

(c) $35-60^{\circ} \mathrm{N}$

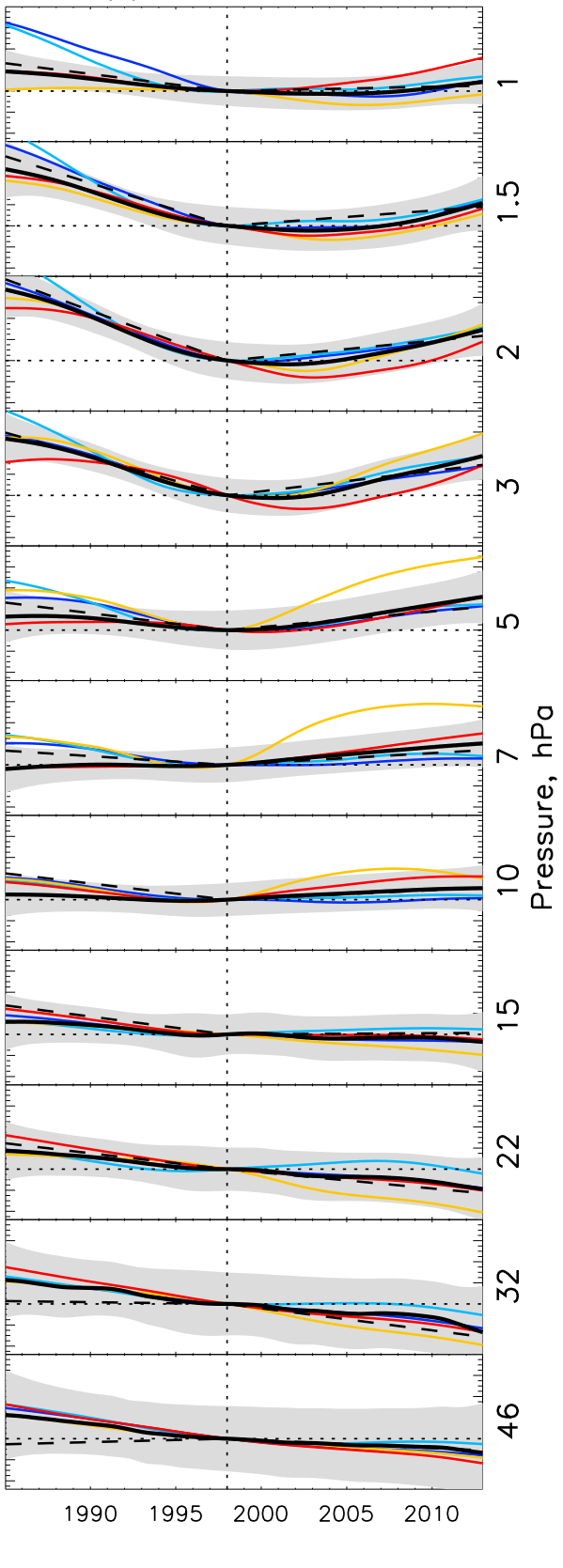

Figure 9. The percentage change in ozone (left axis) relative to 1998 (vertical dashed line; horizontal zero line) for the integrated latitude bands $60-35^{\circ} \mathrm{S}(\mathbf{a}), 20^{\circ} \mathrm{S}-20^{\circ} \mathrm{N}(\mathbf{b})$, and $35-60^{\circ} \mathrm{N}$ (c) and pressure levels from $1 \mathrm{hPa}$ (top; right axis) to $46 \mathrm{hPa}$ (bottom). Only the mean trend lines are shown for GOZCARDS, SWOOSH, SBUV-MER, and SBUV-MOD; the BASIC composite is shown in black with shading representing the $95 \%$ credible interval. The MLR trend estimates for the period before and after January 1998 are given as dashed black lines.

$10 \mathrm{hPa}$ at the Equator and $15-22 \mathrm{hPa}$ in the Southern Hemisphere is very much apparent at the altitudes where DLM and MLR trend estimates disagree on the sign of the trend; this instability of MLR was also noted by Laine et al. (2014) and requires investigation in a future publication to understand. Figure 9 also allows us to observe how the background evolves with time; from this we can see that, while SBUVMER often displays large deviations from the group (e.g. especially at 5 and $7 \mathrm{hPa}$ in all latitude bands), the BASIC composite results are almost always smoothly varying and generally monotonic to/from the years 1998-2002, meaning that a comparison between MLR trends and a change between 
fixed dates from the DLM are indeed valid (the exception possibly being at 1.5 and $1 \mathrm{hPa}$ over the Equator where all datasets display relatively rapid variations in the sign of the DLM gradient, though we note this is where data are more sparse, and temporal sampling can easily be biased by the large diurnal variability; even so, this altitude region appears to be where MLR and DLM are most consistent).

Figure 9 is entirely consistent with, and explains why, Harris et al. (2015) was able to show that the choice of pivot date from the piecewise linear trend using MLR on GOZCARDS led to larger positive trends the later the date of pivot was chosen, i.e. from 1998 to 2002, most prominently above $10 \mathrm{hPa}$ in both midlatitude bands (see Fig. 7 of Harris et al., 2015). We see from the DLM trends in Fig. 9 that at many locations above $10 \mathrm{hPa}$ the gradient is typically zero in 2002 , not 1998 , especially at 3,2 , and $1.5 \mathrm{hPa}$, the exact region where the biggest increase in the trend was found by Harris et al. (2015); northern midlatitude ozone at $1.5 \mathrm{hPa}$ actually appears to start increasing a little later, perhaps in 2004. These results are consistent between all the composites analysed, including the BASIC composite.

It is interesting to note that the two 1998-2012 midlatitude BASIC composite profiles in Fig. 8, while determined independently of each other, display remarkably similar shapes in the DLM analysis, suggesting a symmetry in the stratospheric driving of ozone changes over this period and, indeed, a similar hemispheric recovery following the Montreal Protocol. In contrast, the lower stratospheric mean-profile changes from MLR (dashed black lines in Fig. 8) are not similar, with a generally (and sometimes statistically significant) positive trend in the Southern Hemisphere and (an almost significant) negative trend in the northern midlatitudes.

We propose that the profiles determined by DLM-BASIC are likely to be a better representation of the change in stratospheric ozone than previous estimates. We base this conclusion upon the knowledge that (i) the BASIC approach was successful in identifying and correcting most known artefacts in the ozone composites, (ii) the DLM performed better than the MLR in the artificial ozone time series test cases, and (iii) the DLM-BASIC outperformed both MLR-BASIC and DLM of all the "artefact-damaged" artificial time series. The consistency of independent northern and southern midlatitude DLM profiles for both periods would suggest that additional explanation for why the different hemispheres should evolve in different ways is not required (WMO, 2014). However, this also means that further investigation into why MLR and DLM trend estimates can differ so substantially is needed.

\section{Conclusions}

We have presented a novel approach to identify and account for data artefacts that remain in multiple ozone composites of satellite observations. These artefacts are one of largest remaining causes of disagreement between decadal trend estimates made from the many composites available. Our approach includes estimates of uncertainties using singular value decomposition, a Gaussian-mixture outlier model for the likelihood, and prior information in the form of expected monthly transitions and knowledge of problems in ozone observations; these are combined via Bayesian inference. The main output of this process we term the BAyeSian Integrated and Consolidated (BASIC) composite, which has been designed to account for differences in ozone composites that are constructed in different ways and with observations from different sources. The need for better approaches to combine ozone composites has been raised in recent years as an issue needing resolution (e.g. Tummon et al., 2015; Harris et al., 2015). Harris et al. (2015) stated that it is not currently possible to make definite assumptions about the best way to combine data and in what way, especially when considering multiple composites that use similar, or identical, underlying datasets. Hassler et al. (2014) noted that the key to good estimates of long-term trends is the combination of high-quality measurements and multiple instruments. Our method both requires and benefits from the availability of both. Hassler et al. (2014) further state that the consideration of uncertainties and artefacts is essential, especially when the trends are small compared to the large natural variability (e.g. seasonal cycle), so detailed information is needed about measurement uncertainties, data jumps due to instrument changes, and drifts. Again, our method is specifically designed to address these concerns.

The presence of data gaps, biases between instruments, and issues with sampling, noise, and differences in resolution also enhance uncertainties in trend estimates, which might lead to artificial trends being extracted in multiple linear regression (MLR) analysis. To avoid this, we employed, with refinements, dynamical linear modelling (DLM) (Laine et al., 2014) and found it to be more accurate than MLR when considering test cases where all variance is understood. The combination of the BASIC approach with DLM shows that the problems listed above can indeed be resolved to improve estimates of ozone changes on decadal timescales.

The results presented here are a step forward, but we do not consider the composite a definitive and final product; there are still issues to resolve, which we extensively discuss (Sect. A5.2). These caveats include the concern of using the same instrument dataset more than once, even though it may be used in separate composites with different preprocessing (Harris et al., 2015). Our recommendation to resolve this problem, and as the natural next step forward, is to apply the posterior sampling approach as a method to combine as many independent datasets as possible, integrating all the known caveats and uncertainties. This will require an additional step to the methodology outlined here in order to account for absolute bias between the datasets, but we do not consider that this will cause significant difficulties. 
From the DLM analysis, the estimated changes in ozone between 1985 and 1997, and then between 1998 and 2012, show good agreement with the shape of the ozone profiles presented by Harris et al. (2015), where seven composite datasets were combined with various approaches to estimate errors. The BASIC composite results using DLM (and MLR) show remarkably similar profile shapes and magnitudes for the earlier period. The implication for the latter period, then, is that ozone is indeed clearly and significantly recovering in the upper stratosphere as a result of the Montreal Protocol, which has not previously been demonstrated universally with significance from observations, though Shepherd et al. (2014) demonstrated that the recovery was indeed underway by removing dynamics that interfere with calculating trends using a model with specified dynamics. The largest uncertainty in the estimates of Harris et al. (2015) came from considering instrument drift. Since the BASIC composite has accounted for much of this uncertainty, we can be confident that our smaller uncertainties represent an improvement. Further, the BASIC composite typically rejects outliers inconsistent with other composites, or otherwise inflates uncertainty estimates, leading to our assertion that the estimated uncertainties are probably a reasonable reflection of the uncertainty in the observations. Uncertainties on the decadal trends can be further reduced with additional regressors, in addition to a new composite based upon independent instrument datasets rather than the four composites we considered here.

We will make the BASIC composite available and provide supporting documentation should the composite be updated. The composite is available for public use at https://data.mendeley.com/datasets/2mgx2xzzpk/1 (Alsing and Ball, 2017). In future work, we will extend the latitude and altitude range and time period covered, which should lead to more robust results and an improved assessment of ozone trends in the stratosphere.

Data availability. The BASIC composite is available at https:// data.mendeley.com/datasets/2mgx2xzzpk/1; please cite this publication and the citation for the data page, Alsing and Ball (2017), when using the data. 


\section{Appendix A}

\section{A1 Additional information on SBUV composites}

In the construction of SBUV-MER, ozone was considered in $5^{\circ}$ daily zonal means and was used in regressions over periods of instrument overlap to account for different variability and combine datasets into the composite; this was also used to identify and account for biases. Specific caveats of the SBUV-MER composite include (see also Fig. 1) (i) the NOAA-11-16 overlap is very short, so only a bias offset was applied; (ii) to avoid a propagation of non-physical NOAA-9 trends to the earlier Nimbus-7/NOAA-11 periods, Nimbus-7 and NOAA-11 are not adjusted - this is the major difference between the dataset in Tummon et al. (2015) and the revised dataset used here - only NOAA-9 is adjusted between the two parts of NOAA-11, and NOAA-14 is used as a bridge to the descending part of NOAA-11, but does not appear in the final dataset; (iii) there are large differences in the slope and intercept between 20 and $3 \mathrm{hPa}$, especially with respect to the adjustment of NOAA-14 to NOAA-11 during the 19972000 overlap; (iv) while NOAA-16 and -17 are consistent with respect to SAGE-II instrument observations, the correction approach is not as effective for NOAA-16 and -17 at higher pressures (lower altitudes) at latitudes away from the Equator.

In the construction of SBUV-MOD, Frith et al. (2014) looked at offsets in the total column ozone and showed that instruments typically agreed within the stated uncertainty estimates from Monte Carlo simulations, so no additional offsets were applied to further correct them. Kramarova et al. (2013b) and Labow et al. (2013) had also previously shown that the SBUV total ozone agrees to within $1 \%$ with the ground-based Brewer-Dobson instrument network, lidar, and ozonesondes, and was consistent with SAGE-II and Aura/MLS satellite observations to within 5\%. McPeters et al. (2013) also state that instrument overlaps agree to within $\sim 1 \%$ in the globally integrated $\left(60^{\circ} \mathrm{N}-60^{\circ} \mathrm{S}\right)$ total ozone column (TCO), although vertical profiles from NOAA-9, -11 , and -14 had the biggest non-random differences of around $2.3 \%$ between instruments at $2 \mathrm{hPa}$, related to orbit drift, data gaps, and residual uncertainties, while NOAA-16 and -18 showed differences with standard deviations of $\sim 1.3 \%$. However, despite all of this, it is clear from Fig. 7 of Frith et al. (2014) that they were able to identify offsets in the TCO - these offsets mimic the structure of the offsets between the SBUV composites we show in Fig. 2c, indicating that while small in total column, they are on the order of $5 \%$ in the vertical profile, vary in magnitude and sign throughout the atmosphere, and potentially mask offsets in the integrated column.

Kramarova et al. (2013b) and DeLand et al. (2012) also have shown that the 1994-2000 period is of worse quality than earlier and later periods (Frith et al., 2014); DeLand et al. (2012) recommend that NOAA-9 should not be used, which is why NOAA-14 is used for this period in SBUVMOD, although NOAA-11 drifts from 16:00 to 18:00 LT during the 1994-1995 period, for which NOAA-9 is alternatively used in SBUV-MER. A quality "tier" for the satellites was provided in Frith et al. (2014), which is useful in the compilation of the SBUV TCO Merged Ozone Dataset, with drifts tending to cancel in NOAA-11 and -14 overlaps from 1997 to 2000 in TCO, but this does not reveal the profile uncertainties and drifts. The use of the priors for the BASIC composite was necessary to identify and account for the drifts.

\section{A2 Additional information on the SAGE composites}

Due to the low temporal sampling of SAGE-II (15 sunrise/sunset events per day), as opposed to the $\sim 3500 \mathrm{limb}$ emission profiles per day from Aura/MLS, binning of data in GOZCARDS is done into $10^{\circ}$ latitude averages, and datasets are connected by accounting for biases between dataset overlaps. It should be noted that biases always exist between instruments due to calibration, spatial and temporal sampling, profile resolution, signal variability, or retrieval errors. For example, Toohey et al. (2013) showed that occultation sampling errors with respect to emission measurements could reach $10-15 \%$ at high latitudes when atmospheric variability was large. The processing procedure, which occurs before data are binned into latitudes, attempts to remove outliers and impacts from clouds or aerosols and they do not disregard data arbitrarily or attempt to fill in spatial or temporal gaps. The impact of using SAGE II v6.2 instead of v7.0 is discussed by the GOZCARDS team (Froidevaux et al., 2015), which shows very little systematic differences in number density, but leads to large differences when converted to volume mixing ratio (vmr) with temperature from either NCEP or MERRA (as confirmed by Maycock et al., 2016; McLinden et al., 2009). We note that small drifts of $\sim 0.5 \% \mathrm{yr}^{-1}$ do exist between HALOE, SAGE II, and Aura/MLS (Nair et al., 2012; Kirgis et al., 2013), and Nazaryan and McCormick (2005) and Hubert et al. (2016) suggest that most of the datasets used in GOZCARDS have good stability.

In SWOOSH, basic data prescreening is based on published recommendations from satellite instrument teams. SAGE-II ozone screening follows the recommendations of Wang et al. (2002) to remove aerosol contamination and poor quality retrievals; any profile containing more than $10 \%$ uncertainties between 30 and $50 \mathrm{~km}$ are removed. SWOOSH also applies additional screening for profiles before November 1992 affected by the Mt. Pinatubo eruption, using information from the NO observing channel. Offsets applied to the non-reference instrument data vary only by pressure and latitude but not time, such that if drifts exist they may not be accounted for in SWOOSH and GOZCARDS.

We briefly note (and indicate in Fig. 1) technical details in the construction of the SAGE-based composites: (i) for GOZCARDS, there are no months where SAGE-II 

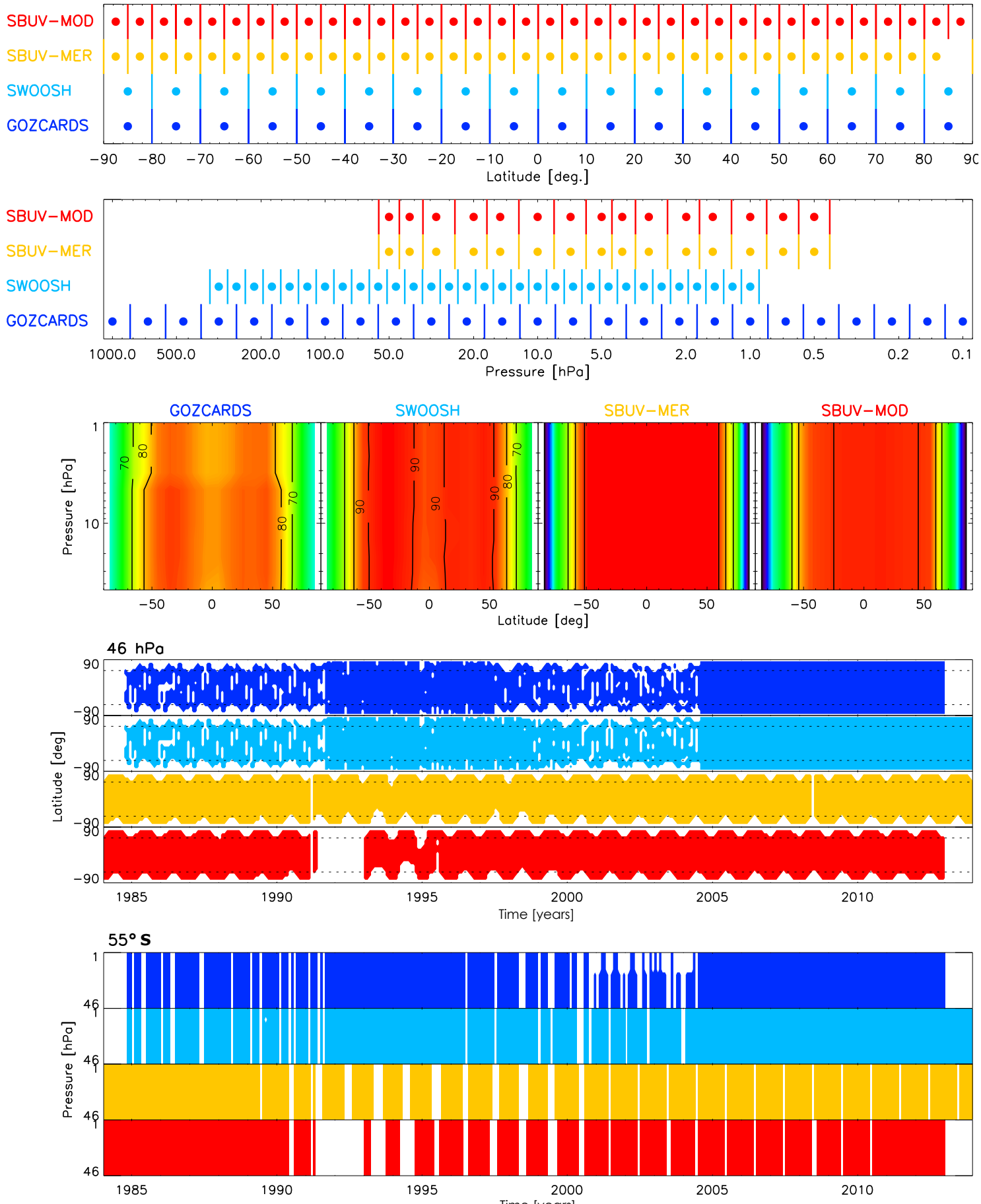

Figure A1. Visual summary of the ozone composites used here. From top to bottom: the latitudinal grid (dots represent the grid centre; lines represent the boundaries); the vertical grid; the percentage of months between 1985 and 2012 where data are available as a function of latitude and pressure level; the data availability as a function of latitude and time at $46 \mathrm{hPa}$; the data availability as a function of pressure level and time at $55^{\circ} \mathrm{S}$. Apart from the third panel, colours are related to each of the composites: GOZCARDS (blue), SWOOSH (light blue), SBUV-MER (yellow), and SBUV-MOD (red).

and ACE-FTS overlap in the NH-tropics due to ACE-FTS coverage being poor; (ii) McLinden et al. (2009) noted that UARS/HALOE and MERRA confirm that there were arte- facts in SAGE-II after 30 June 2000, so these data are not used at altitudes above $3.2 \mathrm{hPa}$; and (iii) problems with the SAGE-II azimuth gimbal in mid-2000, and corrected by 


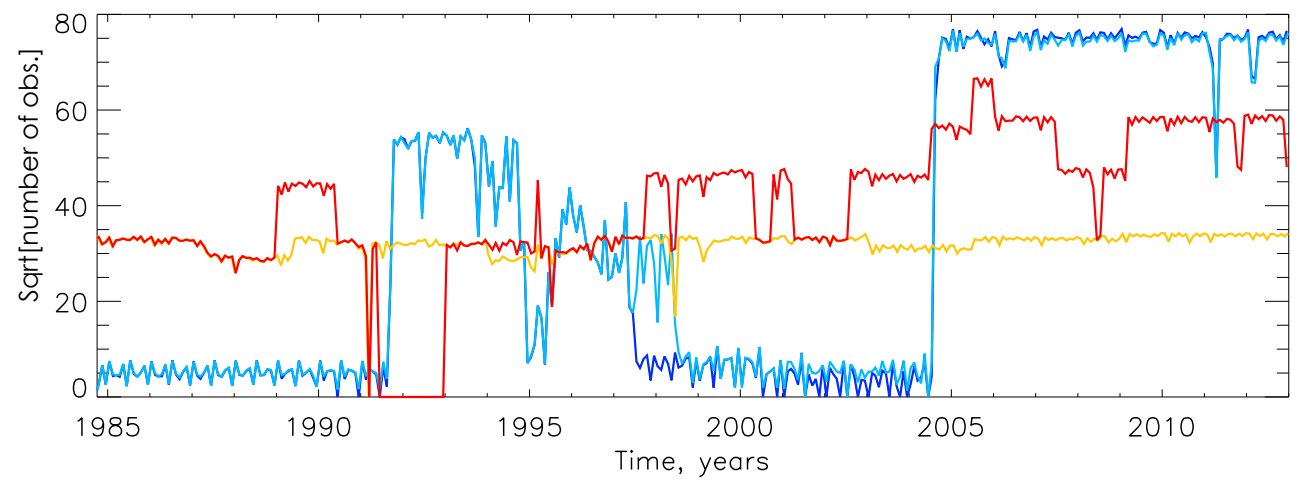

Figure A2. The square root of the latitude-weighted number of observations at $1 \mathrm{hPa}$ between $20^{\circ} \mathrm{S}$ and $20^{\circ} \mathrm{N}$ in each of the composites: GOZCARDS (blue), SWOOSH (light blue), SBUV-MER (yellow), and SBUV-MOD (red).

November, meant there was only a $50 \%$ duty cycle during that period, when it already took about a month to collect data to fully cover latitudes $80^{\circ} \mathrm{S}$ to $80^{\circ} \mathrm{N}$.

\section{A3 Additional information, results, and discussion on the BASIC approach}

\section{A3.1 Effect of the Box-Tiao equation}

In Fig. A3, we show 25 plots for five values of $\gamma$ combined with five values of $\beta$. In this plot, we imagine an idealized scenario of four composites in 1 month with mean values at $-1.5,-1,+1$, and +1.5 , all with an uncertainty of $\sigma=0.2$.

It is clear that for either low values of $\gamma$, and/or low values of $\beta$, we get the expected result assuming all data are independent (which is the dotted line in all plots), but this is inadequate as such a probability density function (pdf) (dotted line/black thick line) does not represent any of the data and is in a region of low probability. For large values of $\beta$ and $\gamma$ (top right), we end up with belief in any of the data points being low (i.e. we enhance $\sigma^{2}$ by a factor of $\gamma^{2}$ ) with any affect from the second (separation) term beginning with $(1-\beta)$ killed off by $\beta \sim 1$; clearly this scenario is not what we are looking for. As the aim is to essentially enhance regions where data agree and reduce belief in outliers, the preferred region of interest is for intermediate values of $\beta(0.1-$ $0.9)$ and $\gamma>10$. From this, we choose $\beta=0.1$ and $\gamma=100$ as this appears to reflect well the desired separation into a multi-modal pdf that represents two independent sets of data (e.g. blue and red/yellow groups).

In terms of its effect on the BASIC composite time series, when combined with a prior expectation, this can lead to the expected time series following one pair (in the example given in Fig. A3) after it has become clear that a jump/offset has occurred, whereas low $\gamma$ or low $\beta$ leads to getting an average of all the composites with a bias introduced by the prior.

\section{A4 Additional information on DLM parameter estimation}

In Figs. A4-A7, we show the recovered posterior distributions for the DLM nuisance parameters $\left\{\sigma_{\text {trend }}, \sigma_{\text {seas }}, \sigma_{\mathrm{AR}}, \rho_{\mathrm{AR}}\right\}$ resulting from the DLM analysis of the BASIC composites performed in Sect. 5. In the case of $\sigma_{\text {trend }}$ (Fig. A4), the posteriors (red) are shown against the applied half-Gaussian priors (blue). In this case, the choice of prior is particularly subjective - in the case where $\sigma_{\text {trend }}$ is allowed to attain large values, the DLM can collapse into a case where the "trend" has so much freedom it can follow the data exactly and capture all variability. Therefore, it is necessary to choose a sensible upper limit on $\sigma_{\text {trend }}$, i.e. on the maximum allowed variability of the smooth background trend. In this study, we chose for the prior on $\sigma_{\text {trend }}$ a half Gaussian, centred on zero, with dispersion 0.0005. All other parameters are given uniform priors.

\section{A5 Success of BASIC approach in accounting for artefacts between composite versions}

BASIC composite results in the main article uses SWOOSH data version 2.6. We originally used version 2.5 (version 2.1 was used by Tummon et al., 2015 and Harris et al., 2015), which was updated to account for an error which led to Aura/MLS being offset in absolute terms by one vertical level. This artefact was clear in our original analysis, and we present an example here to show that the BASIC composite constructed with four composites is relatively unaffected by these types of artefacts.

In Fig. A8, we show the same results for the BASIC composite (black) and SWOOSH version 2.6 (light blue) as in Fig. $6 \mathrm{a}$ and $\mathrm{b}$ at $2.2 \mathrm{hPa}$ and $0-10^{\circ} \mathrm{N}$. In addition, we also show SWOOSH v2.5 (purple) and in red the BASIC composite based on the same input data, but with SWOOSH v2.5 instead of v2.6 ("BASIC(SWv2.5)"). Prior to 2004, the SWOOSH v2.5 line is offset by $\sim+0.3 \mathrm{ppm}$ from the zero line (i.e. relative to BASIC) and SWOOSH v2.6 in Fig. A8b. 


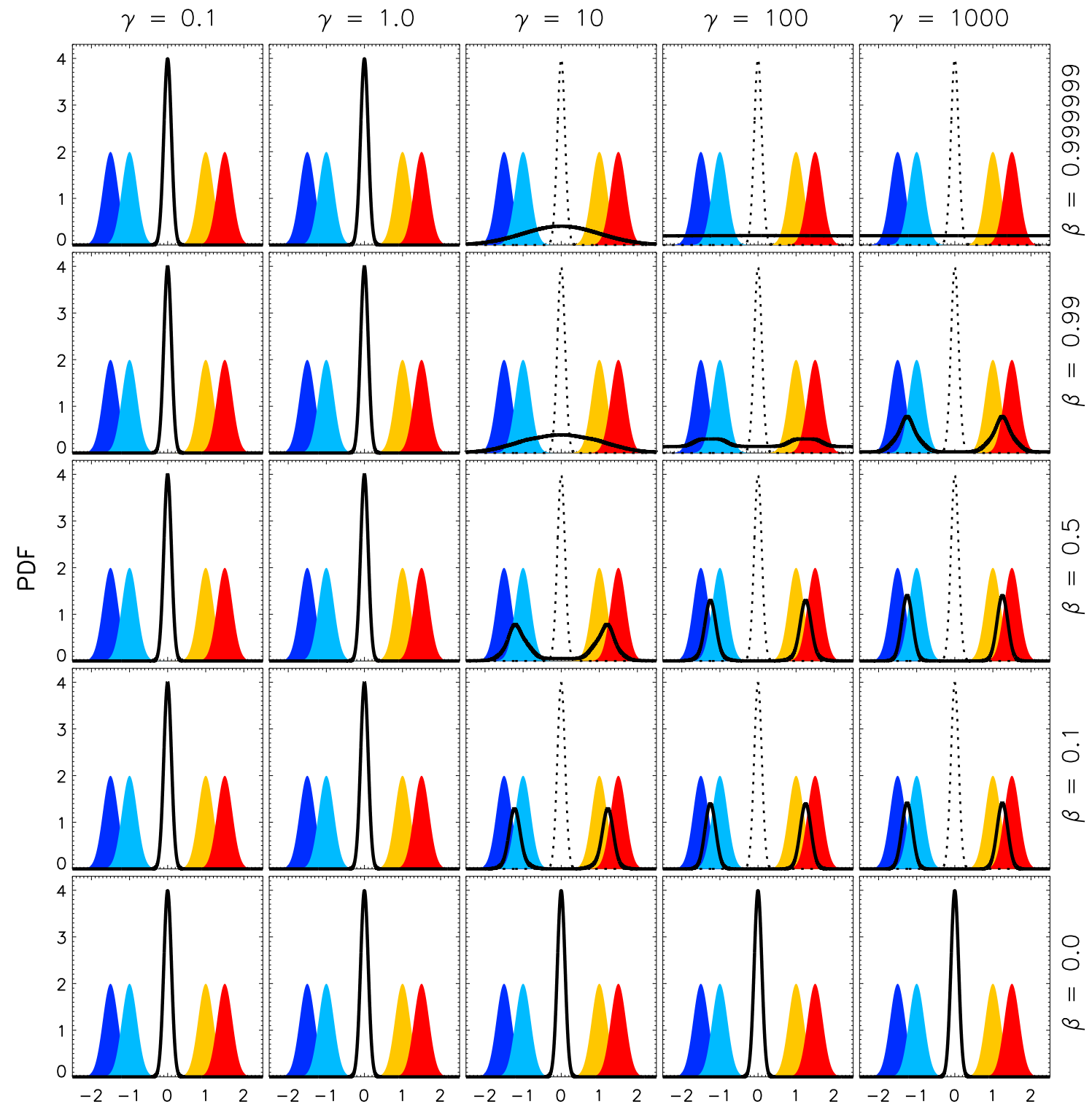

Figure A3. Example of Box-Tiao effect on idealized data with a mean of $-1.5,-1.0,1.0$, and 1.5 (arbitrary units), with blue, cyan, yellow, and red, respectively, all with the same uncertainty $\sigma=0.2$. Dotted lines in all plots represent the pdf resulting from multiplying all data treating them as independent. The solid black line represents the pdf following the Box-Tiao equation. Values of $\gamma$ and $\beta$ used in the Box-Tiao equation in each plot are shown along the upper and right axes.

While there are small variations in the BASIC(SWv2.5) (red line), it also sits close to the zero line, typically with an offset of $\sim+0.05 \mathrm{ppm}$ and ranges between zero and $\sim+0.1 \mathrm{ppm}$. We find that the BASIC composite is similarly unaffected by offsets in the previous version of SWOOSH at other locations.

This example gives us further confidence that when multiple composites are available, the BASIC approach does a good job of accounting for artefacts that exist in only one dataset.

\section{A5.1 Test of BASIC approach using artificial time series}

Given that we do not have any certain measurements against which to test our approach, we need to demonstrate how the BASIC approach operates in ideal, known conditions by using artificial test cases where all the variance is understood. With that in mind, we designed three sets of tests; we present one here and consider DLM and MLR analysis on the other two in Sect. A6.

To create test cases, we took a real ozone time series and from that estimated the regression coefficients of solar, 


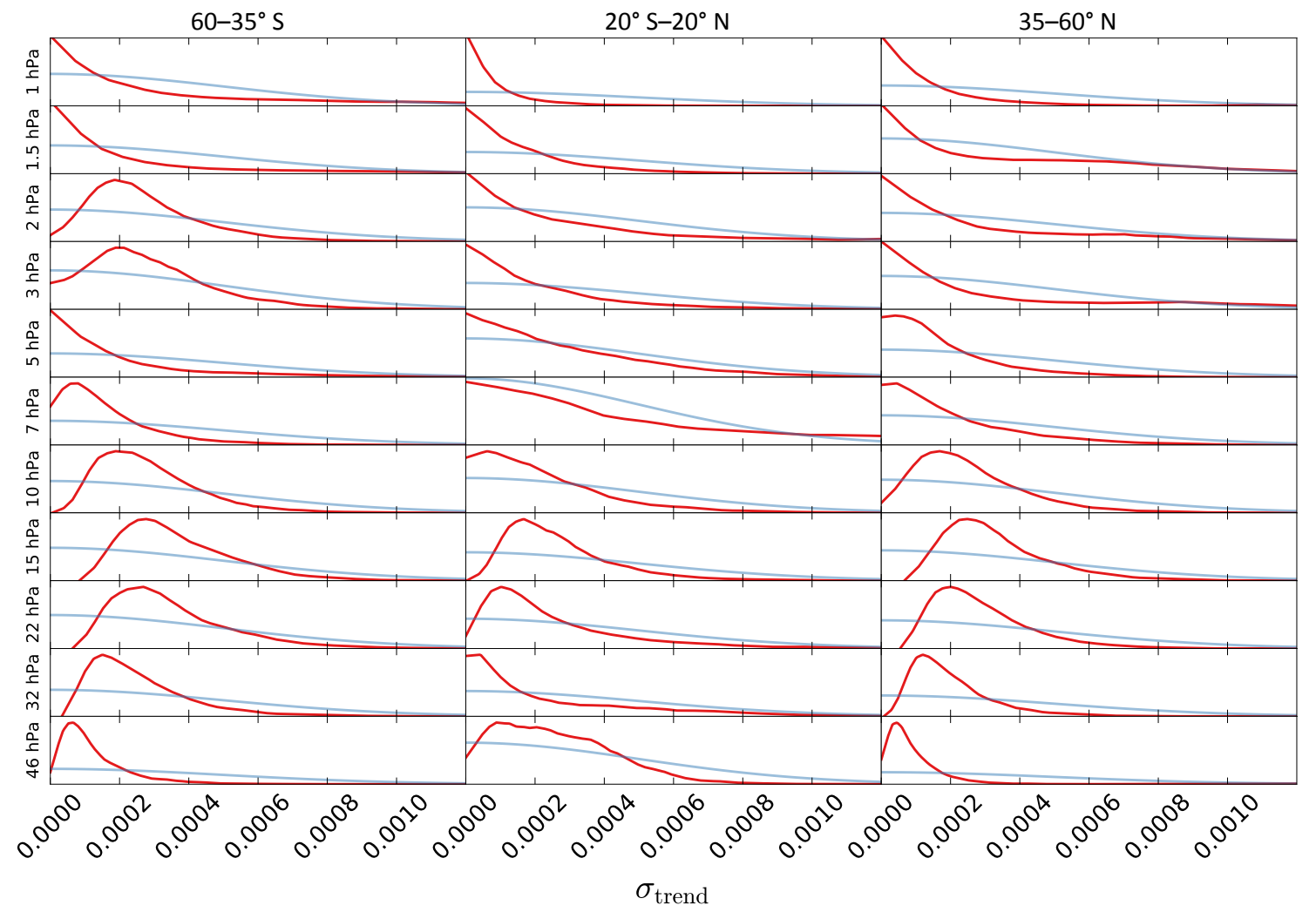

Figure A4. Recovered posteriors on $\sigma_{\text {trend }}$ (red) and the chosen half-Gaussian prior with dispersion 0.0005 (blue) from the DLM analysis performed in Sect. 5 .

ENSO, volcanic aerosols, and two QBO terms using MLR (as in Sect. 5.1), and then reconstructed the ozone time series with these known regressor coefficients, in addition to a realistic seasonal cycle based upon similar variability in the observations. We add a Gaussian noise term but drop unknown residual variance. To represent instrument artefacts and drifts similar to the situation we have here with the SAGE and SBUV composite pairs, we produce artefact time series that are different between pairs, with some other differences within the pairs - these are shown in Fig. A9b as the straight lines. We add these, with different realizations of Gaussian noise for each "instrument", to the artificial time series to produce the "damaged" ozone time series shown in light blue, blue, red, and yellow in Fig. A9a. We then proceed by applying the BASIC approach to the four "damaged" time series exactly as with the real ozone time series/composites; the result is shown in black with the $95 \%$ credible interval in Fig. A9a. The difference of the four artificial time series, relative to the undamaged ozone time series (not shown), are shown in Fig. A9b.

We specifically built the artefact time series to provide difficulties for the BASIC approach. For example, in Fig. A9b at around month 50, all the damaged time series disagree with the undamaged, target ozone time series in the same direction to show that the BASIC algorithm is unable to re- produce the undamaged ozone time series if none of the observations/composites correctly represent ozone during this period. Thus, if all observations are wrong, there is nothing that can be done to resolve the issue other than modelling using, e.g. a chemistry climate model. After month 250, all the datasets are the same (i.e. there are no artefacts except the Gaussian noise that simulates instrument noise and preprocessing differences) and the BASIC approach naturally matches the artificial time series during this period. Prior to month 170 , only one pair is either drifting or has a jump, but not both at the same time, though they are all typically offset from the target: during this period, except when all four are different from the target ( $\sim$ month 50$)$, the BASIC result generally matches the expected ozone within the $95 \%$ credible interval. The period between months 170 and 210 was designed to be complex, with drifts and jumps occurring within and between pairs in rapid succession. The BASIC result, unsurprisingly, does not perform so well during this period though it does not generally deviate too far from the target; between 200 and 250, it is closer to one pair, but sits between all four since there is roughly equal information and uncertainty in each of them. Throughout, when the artificial time series are far apart, the BASIC result uncertainties typically increase to accommodate the higher uncertainty. 


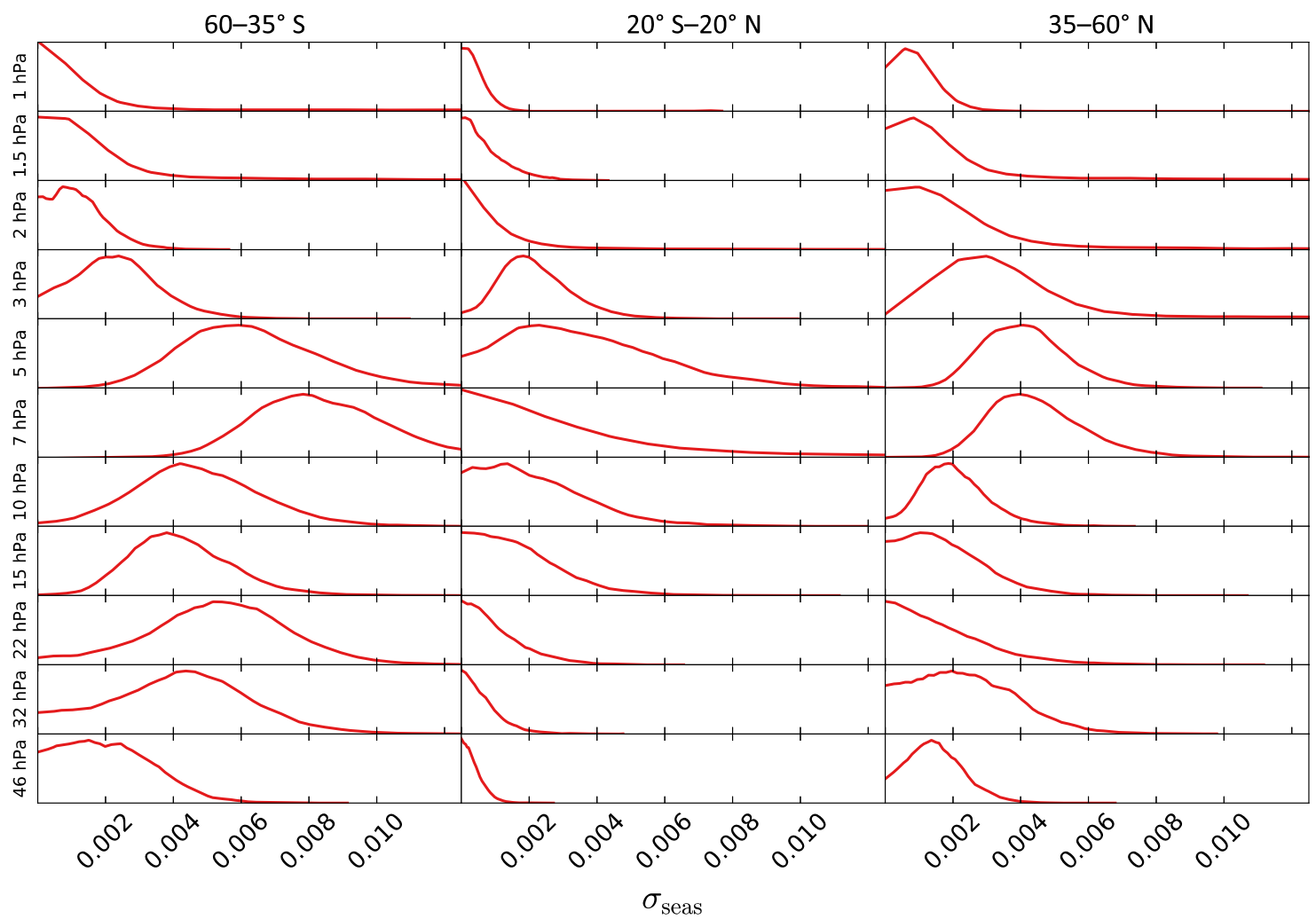

Figure A5. Recovered posteriors on $\sigma_{\text {seas }}$ from the DLM analysis performed in Sect. 5.

\section{A5.2 Caveats on using the BASIC approach}

So far, we have discussed several drawbacks with the current version of the BASIC approach presented here. Here, we collate and list these, and briefly discuss potential solutions for the future, where available.

1. Vertical resolution: This is a problem related to the different averaging kernels of the various instruments used to construct the composites - the SAGE composites use instruments that all have higher resolution than those in the SBUV composites. This difference in vertical resolution becomes more important at lower altitudes, and it is clear in the case of the QBO signal being different (Bhartia et al., 2013). Kramarova et al. (2013a) recommends only using the integrated column from SBUV data below $25 \mathrm{hPa}\left(16 \mathrm{hPa}\right.$ between $\left.\pm 20^{\circ}\right)$, because although SBUV is sensitive to ozone in the troposphere and lower stratosphere, the vertical distribution of that ozone is determined by a priori constraints. Alternatively, when making direct comparisons between SBUV and other high vertical resolution instruments (e.g. Aura/MLS), Bhartia et al. (2013) advise using the SBUV kernels to degrade the resolution of the instrument to match the vertical resolution of SBUV before comparing. However, given that some issues with resolution are already evident at $10 \mathrm{hPa}$ (Fig. 6) and that there is still some useful information in the ozone observations below $25 \mathrm{hPa}$, we still consider the data relevant in this study. This issue should not represent a significant problem when MLR or DLM analyses are performed since the two QBO regressor terms should capture much of the QBO variability. However, if one is interested in the QBO itself, then we would also recommend using the SAGE-based composites and/or datasets used to construct them (see also Kramarova et al., 2013a). We would not endorse a solution based on de-weighting a composite relative to its vertical resolution, because then SBUV will always be at a lower weight than the SAGE composites and the BASIC approach will always favour the latter.

2. Double counting: The use of only two pairs of composites, each built using the same underlying instrument data, resolves one of the concerns of Harris et al. (2015) about biasing our result towards the composites with the most common instrument data (e.g. five of the seven composites combined by Harris et al., 2015 used SAGE-II as a major component). However, this leads to the problem that for periods when two of the composites are identical (i.e. not offset and with similar artefacts), the likelihood estimate may be biased in favour of that pair, which are being treated as independent datasets when indeed they are not. An example can be seen in 


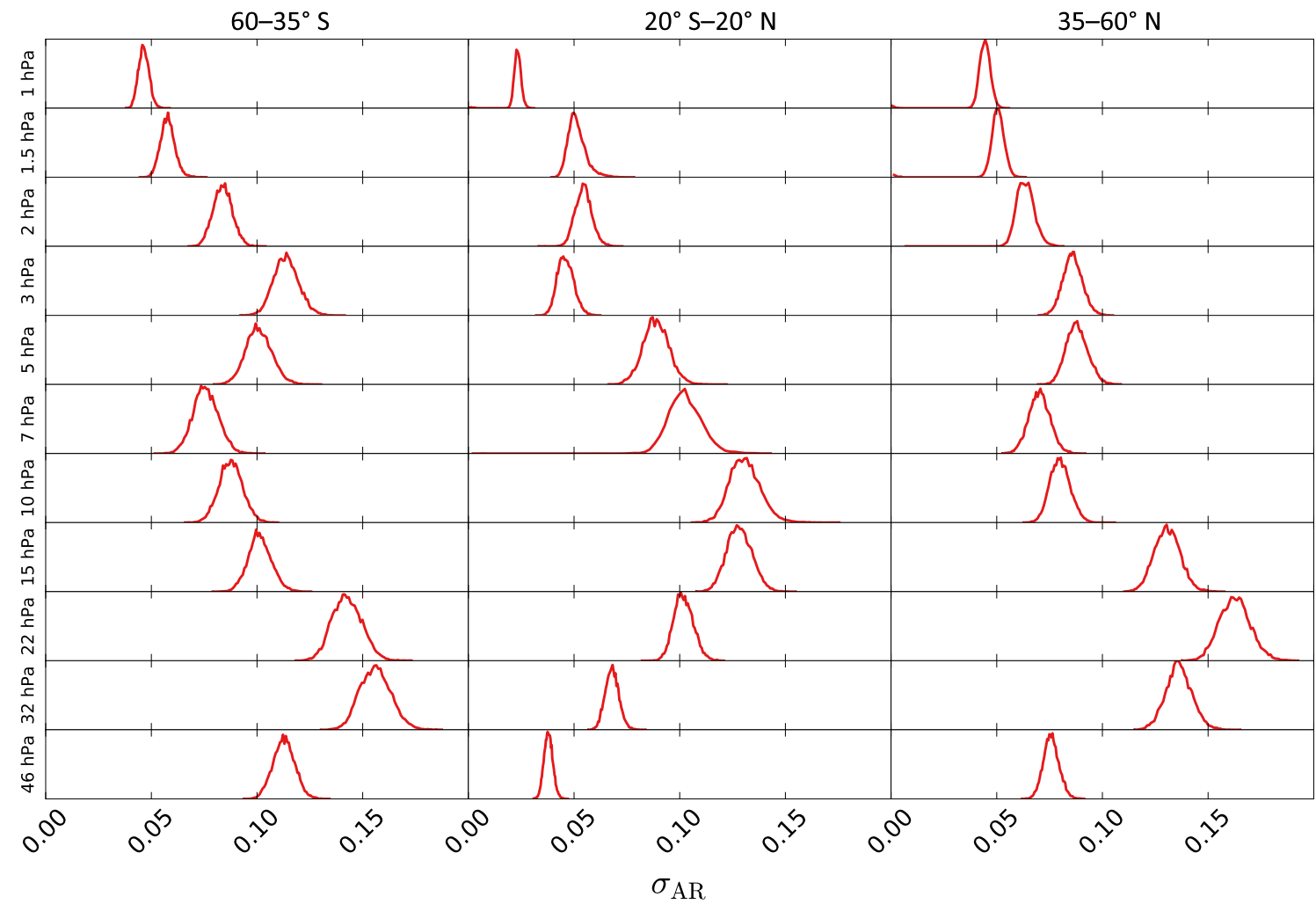

Figure A6. Recovered posteriors on $\sigma_{\mathrm{AR}}$ from the DLM analysis performed in Sect. 5 .

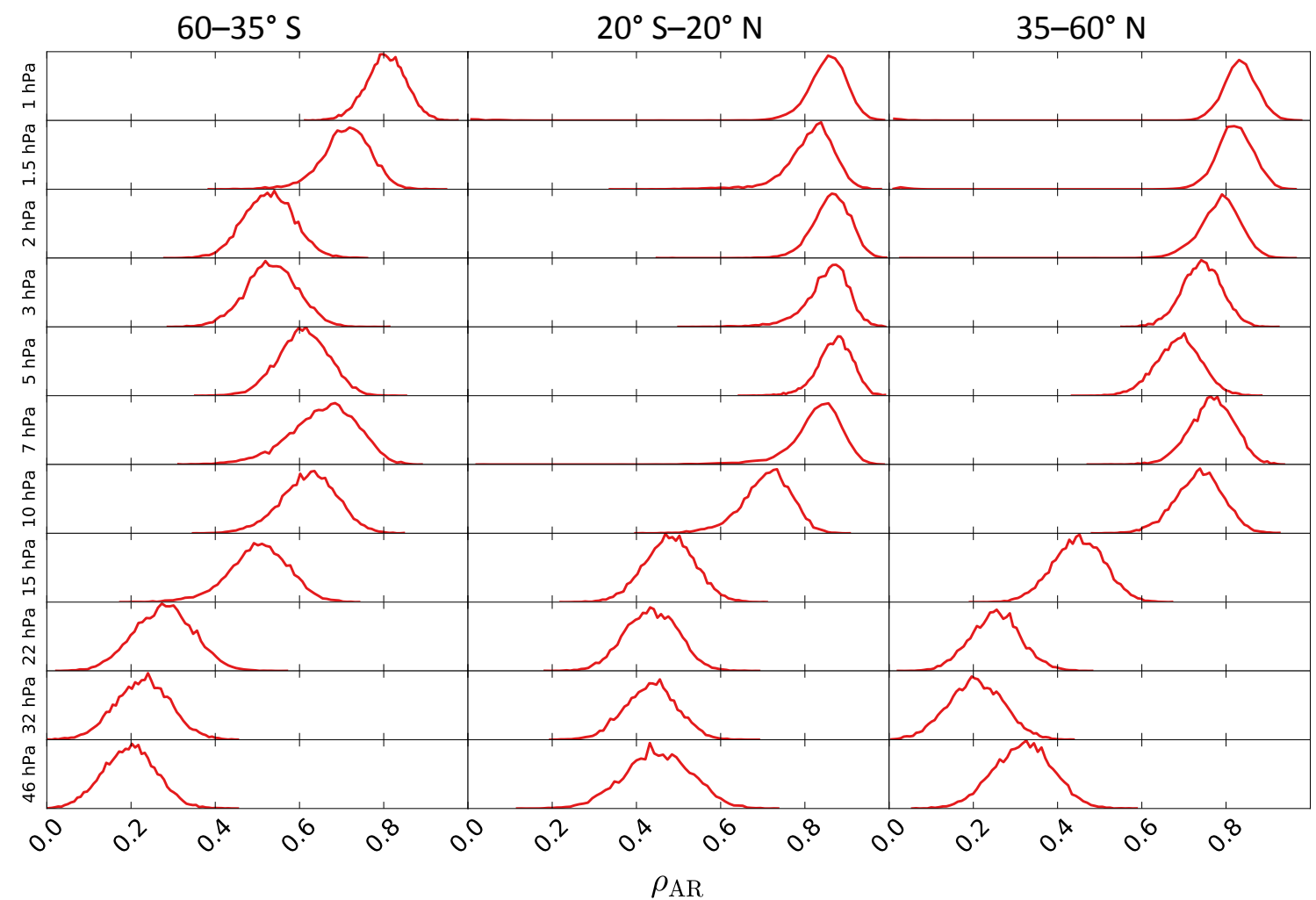

Figure A7. Recovered posteriors on $\rho_{\mathrm{AR}}$ from the DLM analysis performed in Sect. 5. 


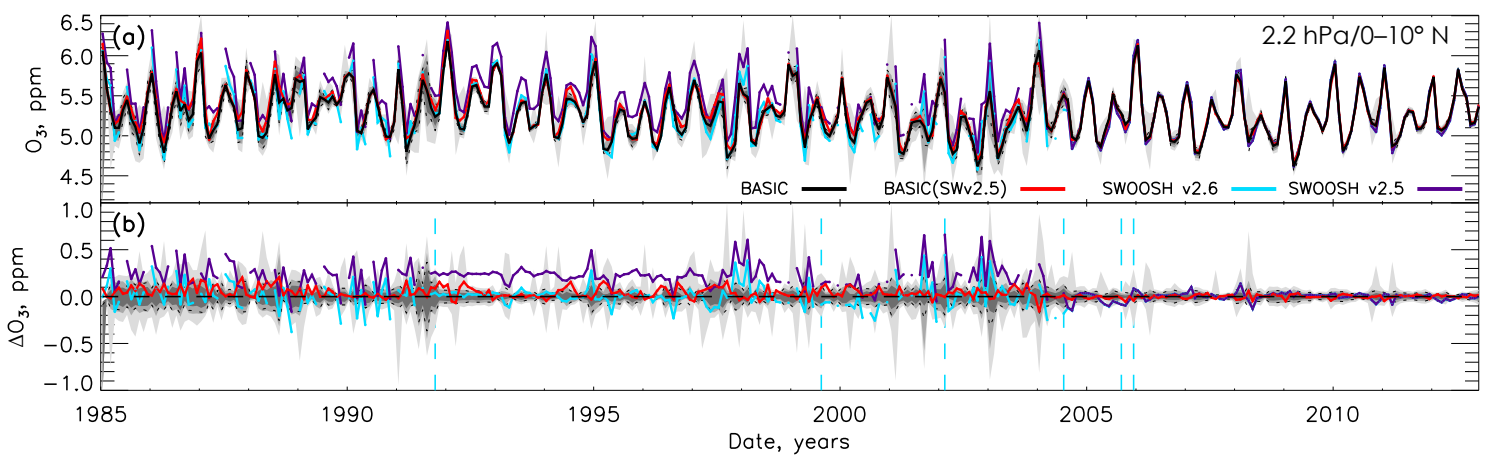

Figure A8. Ozone time series from 1985 to 2012, all bias shifted to the mean of SWOOSH v2.6 after August 2005. (a) Absolute ozone at $2.2 \mathrm{hPa}$ over $0-10^{\circ} \mathrm{N}$ from SWOOSH v2.6 (light blue), SWOOSH v2.5 (purple), BASIC using SWOOSH v2.5 (red), and the BASIC composite using SWOOSH v2.6 (black, with shading representing $68 \%$ (dark grey), $95 \%$ (grey), and 99\% (light grey) credible intervals (CIs), and 2 standard deviations (dotted lines)). Panel (b) is the same as (a) but for the difference relative to BASIC (SWOOSHv2.6).

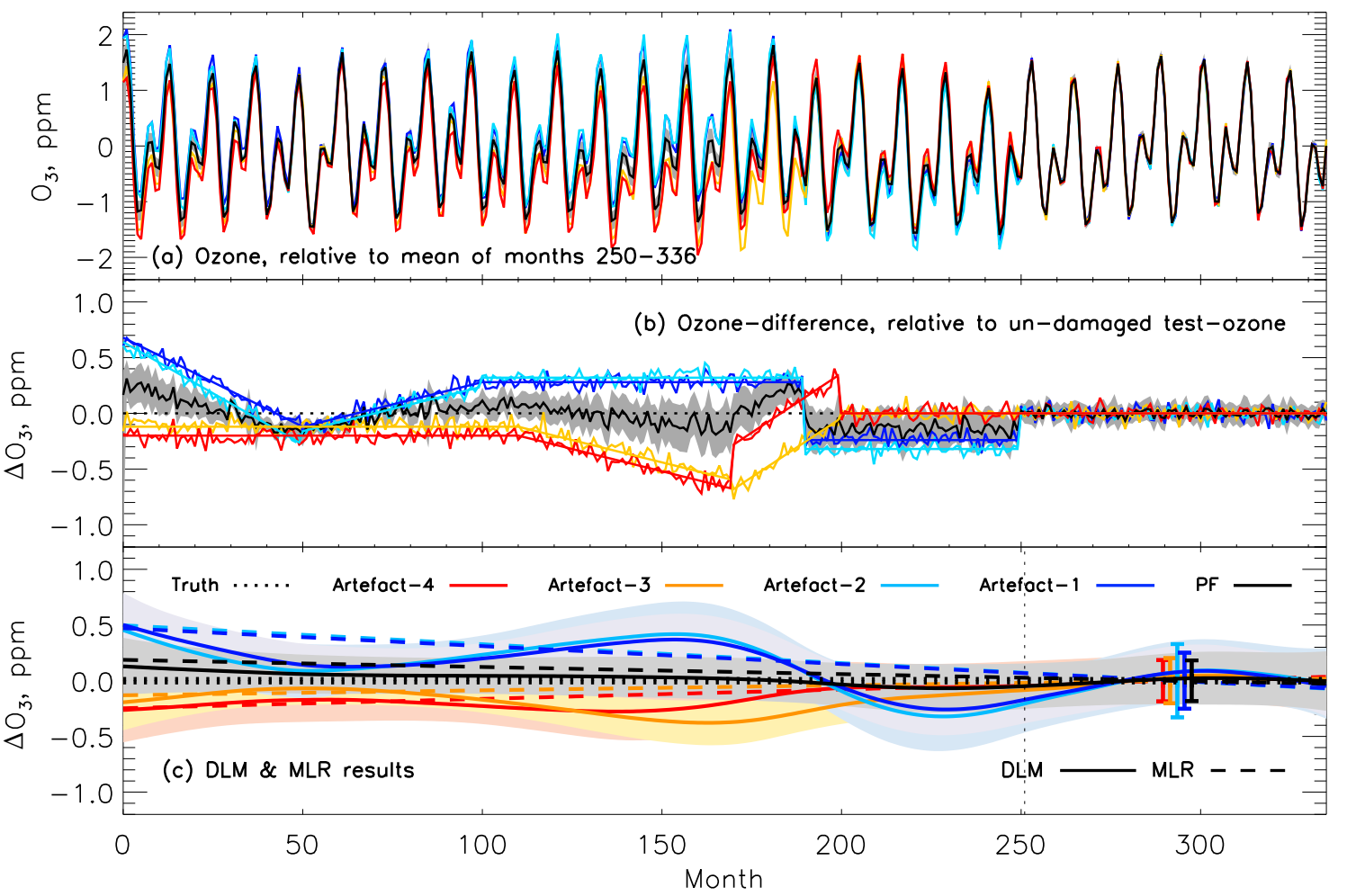

Figure A9. A test case to evaluate the performance of the BASIC approach. Damaged time series are plotted in panel (a) relative to the mean of months after 250 in light blue, blue, yellow, and red, and the BASIC result in black. Differences of time series in panel (a) relative to the undamaged (test) time series is shown in panel (b); the straight coloured lines in panel (b) represent the artefacts applied to the undamaged time series to produce the damaged ones in panel (a); grey and shading in panels (a) and (b) represent the $95 \%$ credible intervals of the BASIC result. In panel (c), we show the estimated trends over the full period from multiple linear regression (MLR; dashed) and the dynamical linear model in solid lines. The true trend during this period is zero (dotted line).

Fig. 6 b prior to 1991 , where the SAGE composites are offset from each other, but the SBUV composites are at almost identical levels. It is fortuitous that the level of SBUV is in close agreement with SWOOSH before and after 1991, but this may not be the case in other locations. In reality, we should not treat the SBUV data as independent during this early period, but this adds further complications in making decisions about when they should be considered independent or not. We choose not to make this decision as this removes much of the objectivity that the BASIC approach provides. To account for this in the future, we recommend that the approaches put 


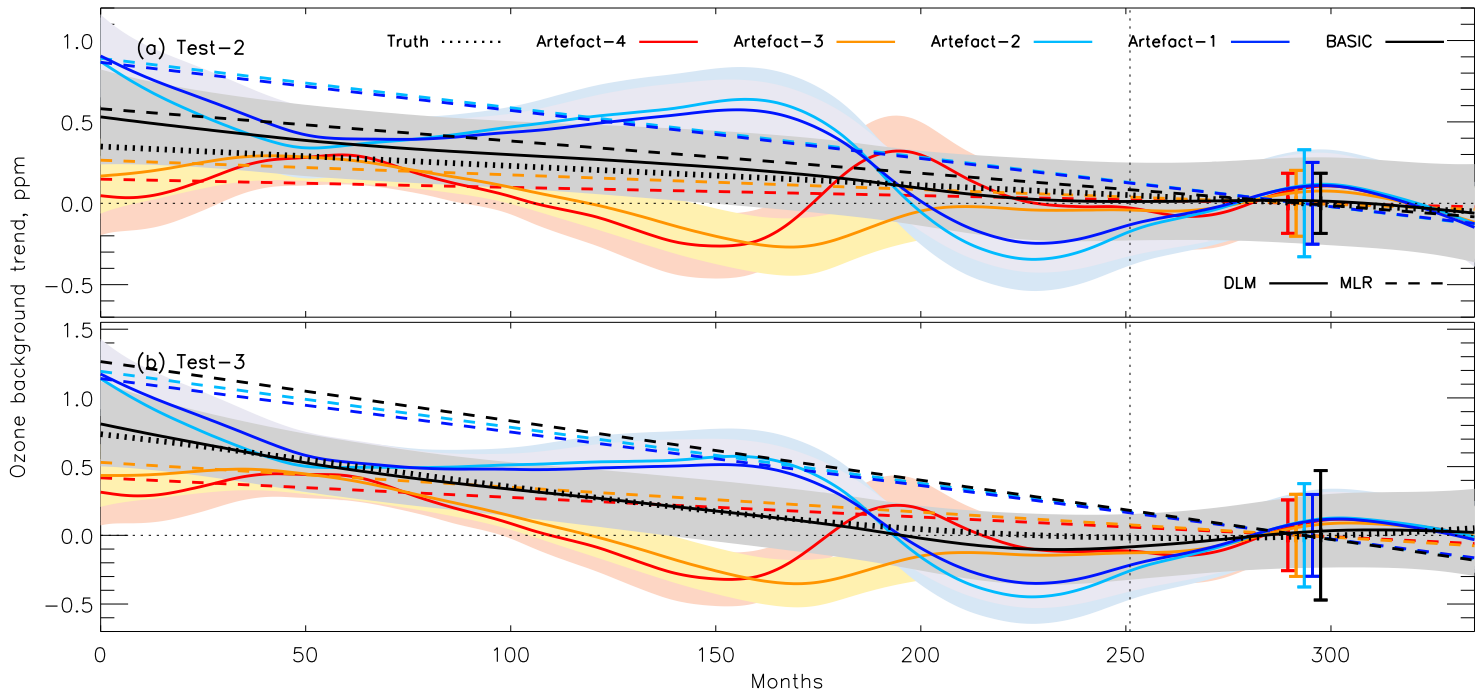

Figure A10. Similar to Fig. A9c: two additional tests cases where the only change is that the background trend in panel (a) is linear and in panel (b) non-linear, as shown with the thick, dotted black line.
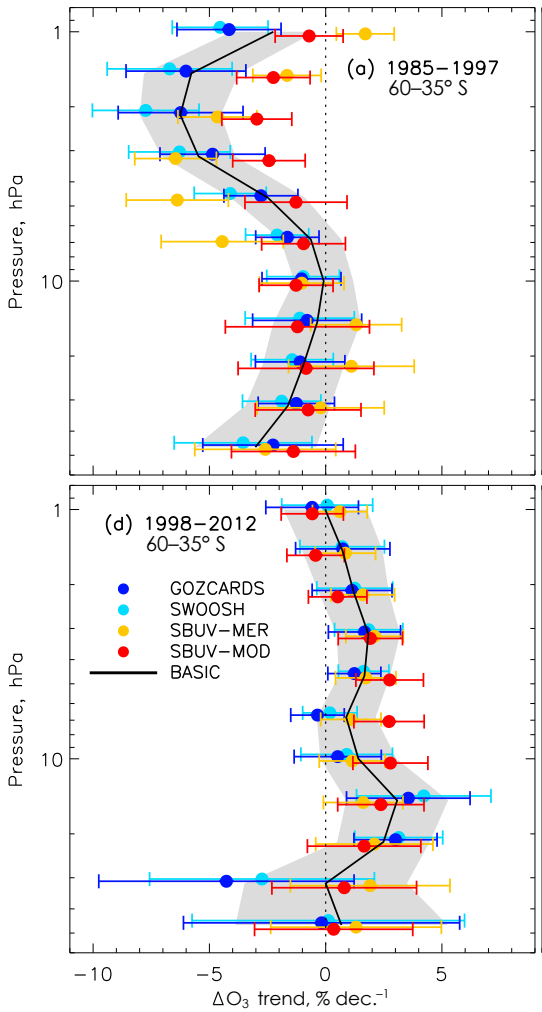

(b)
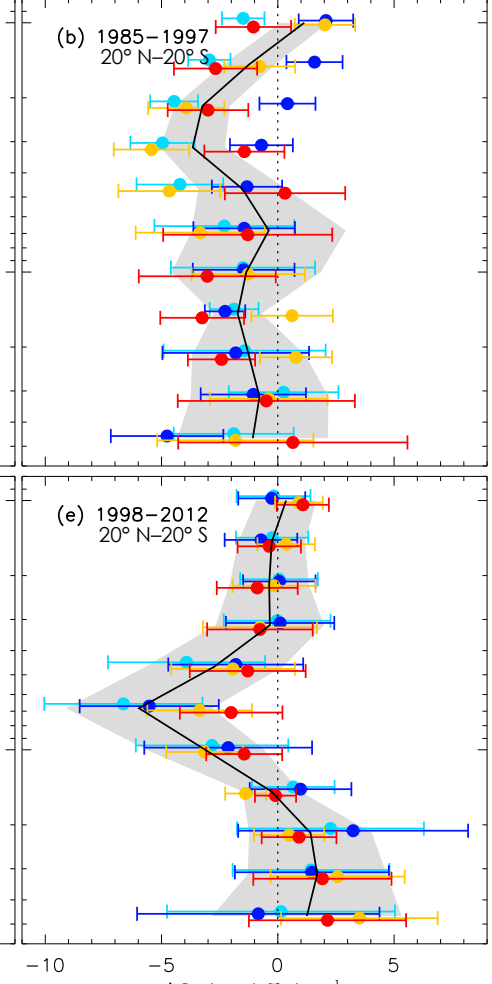
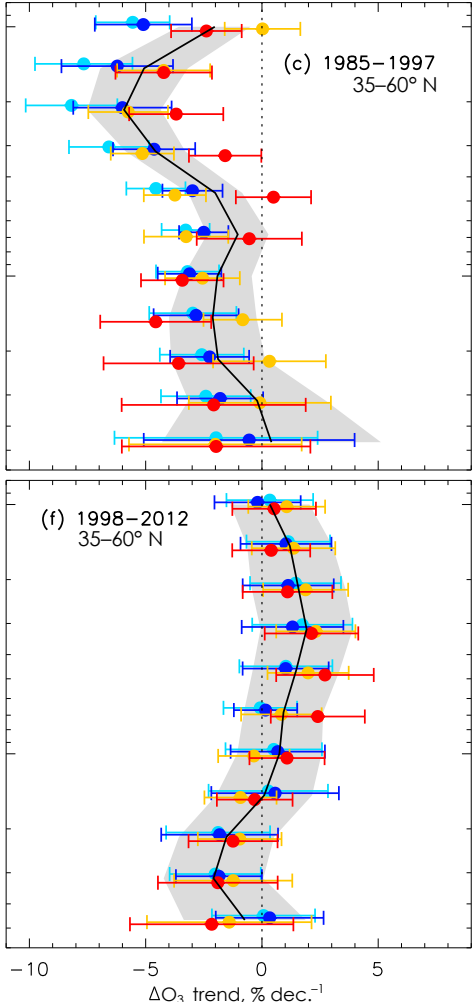

Figure A11. The decadal trend in ozone from multiple linear regression (MLR) between 1985 and 1997 (a-c) and 1998 and 2012 (d-f), over $60-35^{\circ} \mathrm{S}(\mathbf{a}, \mathbf{d}), 20^{\circ} \mathrm{S}-20^{\circ} \mathrm{N}(\mathbf{b}, \mathbf{e})$, and $35-60^{\circ} \mathrm{N}(\mathbf{c}, \mathbf{f})$. GOZCARDS, SWOOSH, SBUV-MER, and SBUV-MOD are shown with $95 \%$ credible intervals; the BASIC composite is shown in black with shading representing $95 \%$ credible intervals.

forward here should be applied to the original datasets underlying the composites, each considered independently but with prior information, to construct a composite. This would require an additional step to estimate the offset between datasets, and to assign one dataset as a reference, but this would be a relatively straightforward addition to the procedure. 
3. Restricted altitude range: We currently only consider the pressure range $47-1 \mathrm{hPa}(\sim 20-48 \mathrm{~km})$ as we are restricted to those covered by all the composites. The GOZCARDS and SBUV composites go higher, but observations in this region are subject to rapid diurnal changes that require good geolocation and temporal sampling, and the local time of the observations must be taken into account. MLR trend analysis (Fig. A11) shows that the composites can display significant, different long-term behaviour at 1.5 and $1 \mathrm{hPa}$, even between pairs of composites (though this is less the case using DLM in Fig. 8); this is also where diurnal variability is a serious issue, as mentioned by all groups in either publications or user documentation (e.g. see McPeters et al., 2013; Davis et al., 2016 and references therein). This is an issue that is still being investigated by the community, and we do not address it here. However, in addition to prescreened data, it may be something that is possible to resolve with accurate transition priors, and additional prior information, in addition to the ones we already suggest using here. Observations are also available down to $316 \mathrm{hPa}$, but there are large gradients in ozone at these levels, so even the relatively high resolution of the instruments in the SAGE composites can struggle to accurately resolve variability at individual layers this low down. However, many observations do exist, and so when integrating the original data using the BASIC approach (see previous point), these layers could be included, and additional prior information could also be used to account for the large ozone gradients.

4. Restricted latitude range: While the composites extend to higher latitudes than $60^{\circ}$, at these latitudes the need for direct or scattered sunlight leads to several months of the year where data are missing, with increasing periods of the year without observations closer to the poles. We do not attempt to fill these data without observations available. In the future, we could use night-viewing instruments such as GOMOS (Kyrölä et al., 2013) to extend into higher latitudes when these data are available (i.e. after 2002), but it is not possible to do it prior to the GOMOS measurements, except potentially through ground-based observations, though they are usually limited to lower altitudes than the satellite observations can consider. In the future, we could also consider extending the BASIC approach to better estimate ozone during at least the summer seasons.

5. Mt. Pinatubo: The example given at $10 \mathrm{hPa}$, and checks at other locations, clearly indicates that the BASIC approach is able to avoid the artificial decrease in the SBUV-MER data between June 1991 and 1992. Frith et al. (2014) advise caution when using data in the 69 months following the eruption, especially for $15^{\circ} \mathrm{S}-$ $30^{\circ} \mathrm{N}$. Thus, for this altitude, when using MLR to anal- yse trends, we also advise caution during this period because the SAGE pair dominate during this period, and if Pinatubo-eruption-related artefacts remain in these data, they will influence the BASIC composite during this time. One idea to consider would be to increase the prior de-weighting factor over this period, but this would be an additional subjective decision, so we prefer to flag this information instead and find a more elegant solution in the future. However, such a problem may not be possible to resolve if the eruption inherently affects observations which cannot be removed prior to applying the BASIC algorithm.

Some of these caveats may be resolved with additional information from the ozone community and by using the BASIC approach to construct a composite from the original, individual instrument time series. Nevertheless, for the work involving composites here, we conclude that despite these issues, overall the BASIC approach performs well in estimating ozone variability. This conclusion is based upon the artificial test case target time series being well estimated, the results of the example real ozone time series presented in Fig. 6 that account for known issues, and the success in the case of the SWOOSH version changes where the BASIC approach accounts for the problems in SWOOSH in v2.5 in advance of the v2.6 release (Sect. A5).

\section{A6 Comparison of multiple linear regression and dynamical linear modelling in estimating long-term trends}

To test the ability of MLR and DLM to estimate the background trend, we use the artificial test cases presented in Sect. A5.1 and Fig. A9a, in addition to two more with the same regressor coefficients and noise, but with linear and non-linear time-varying background trends (Fig. A10). The first set has a background, linear, zero trend (Fig. A9c), the second a linear downward trend (Fig. A10a), and the third a downward-linear trend plus a non-linear curve that reaches a minimum in the latter half of the full period before increasing again (Fig. A10b); the true "target" trends are shown in Figs. A9c and A10a and b as thick, dotted black lines. In each case, we apply the BASIC approach to the four sets of artefact-damaged time series, as in Fig. A9. Therefore, we have 15 test time series, all fully understood. This does not represent the situation for the real ozone time series since in many of those cases the MLR residuals (unaccounted for variability) can typically account for $\sim 50 \%$ of the variance. However, these tests with artificial ozone time series are indicative of the performance with real time series.

One major advantage of DLM over MLR for estimating long-term trends is that MLR requires the trend to be prescribed in advance as linear, or piecewise linear trends (e.g. Kyrölä et al., 2013), or is expected to follow the equivalent stratospheric chlorine (EESC) curve; (Newman et al., 2001). 
The shape of the EESC, which follows CFC stratospheric loading that peaked in the mid-to-late 1990s, impacts more on the sensitivity of the MLR analysis than the period length does when calculating decadal trends (WMO, 2011). The main problem in assuming an EESC shape is that the timing of chlorine minimum is location dependent with, e.g. higher latitudes lagging those closer to the Equator since it takes time for chlorine changes to reach different regions. Therefore, fixing the decline date may lead to misleading estimates (Harris et al., 2015). The use of the DLM allows this issue to be circumvented to some degree by not fixing the background trend or an inversion date (Laine et al., 2014) and allowing it to vary with time, though this still does not necessarily separate EESC from dynamical changes related to, e.g. changes in the BDC (Polvani et al., 2011; Harris et al., 2015).

In Fig. A9c, we plot the MLR (dashed) and DLM (solid) trend results ${ }^{4}$. In this example, the true long-term trend is zero (dotted black line). The only result that is able to stay within 2 standard deviations of the "truth" is the DLM of the BASIC result, and usually it is within 1 standard deviation. The MLR of the BASIC result shows a significant downward trend, and naturally one would not expect the MLR of the damaged time series to estimate an accurate result. What is interesting to observe is that the DLM accurately extracts the drifts in the damaged background trends as well, which might be useful in future studies to further assess anomalous behaviour in the composites by interpreting the behaviour of the DLM results from each composite. The two tests with the linear and non-linear background trends (Fig. A10) can lead to essentially the same conclusions, with the non-linear trend being fitted almost exactly, while MLR is significantly off from the "truth". A more thorough assessment of the DLM with respect to MLR will be made in a forthcoming publication.

In summary, our tests suggest that when estimating the long-term trend, the use of the BASIC approach to correct data, together with the DLM, is more successful and accurate than using MLR or DLM on uncorrected time series. Therefore, we would recommend using the BASIC approach combined together with the DLM for the analysis of longterm trends in ozone, as outlined in this study.

\footnotetext{
${ }^{4}$ Note that in these test cases for our DLM inference we assume a half-Gaussian prior on $\sigma_{\text {trend }}$ with dispersion 0.001 rather than 0.0005 . This is for illustrative purposes, to emphasize the impact of "damaging" the time series on the recovered trend, and we note that the choice of prior on $\sigma_{\text {trend }}$ is in any case subjective (see Sect. A4).
} 
Competing interests. The authors declare that they have no conflict of interest.

Acknowledgements. We thank the referees, Daan Hubert and Marko Laine, for their thorough input which led to significant but important changes that strengthened the paper as a result. We thank Stacey Frith, Jeannette Wild, and Lucien Froidevaux for detailed comments on the composite datasets and general comments on the manuscript. We also thank the GOZCARDS, SWOOSH, SBUV-MOD, and SBUV Merged Cohesive composite teams for use of their data. William T. Ball and Eugene V. Rozanov were funded by Swiss National Science Foundation (SNSF) grant 200020_163206 (SIMA). Fiona Tummon was funded by SNSF grant 20F121_138017. GOZCARDS ozone data can be found at https://gozcards.jpl.nasa.gov/. SWOOSH ozone data can be found at http://www.esrl.noaa.gov/csd/groups/csd8/swoosh/. SBUV ozone data can be found at http://acd-ext.gsfc.nasa.gov/Data services/merged/.

Edited by: Bernd Funke

Reviewed by: Daan Hubert and Marko Laine

\section{References}

Adams, C., Bourassa, A. E., Sofieva, V., Froidevaux, L., McLinden, C. A., Hubert, D., Lambert, J.-C., Sioris, C. E., and Degenstein, D. A.: Assessment of Odin-OSIRIS ozone measurements from 2001 to the present using MLS, GOMOS, and ozonesondes, Atmos. Meas. Tech., 7, 49-64, https://doi.org/10.5194/amt-7-492014, 2014.

Alsing, J. and Ball, W. T.: BASIC Composite Ozone Time-Series Data, Mendeley data, https://doi.org/10.17632/2mgx2xzzpk.1, 2017.

Arnold, S. R., Methven, J., Evans, M. J., Chipperfield, M. P., Lewis, A. C., Hopkins, J. R., McQuaid, J. B., Watson, N., Purvis, R. M., Lee, J. D., Atlas, E. L., Blake, D. R., and Rappenglück, B.: Statistical inference of $\mathrm{OH}$ concentrations and air mass dilution rates from successive observations of nonmethane hydrocarbons in single air masses, J. Geophys. Res.-Atmos., 112, D10S40, https://doi.org/10.1029/2006JD007594, 2007.

Bhartia, P. K., McPeters, R. D., Flynn, L. E., Taylor, S., Kramarova, N. A., Frith, S., Fisher, B., and DeLand, M.: Solar Backscatter UV (SBUV) total ozone and profile algorithm, Atmos. Meas. Tech., 6, 2533-2548, https://doi.org/10.5194/amt-6-2533-2013, 2013.

Box, G. E. P. and Tiao, G. C.: A Bayesian Aapproach to some outlier problems, Biometrika, 55, 119-129, https://doi.org/10.1093/biomet/55.1.119, 1968.

Carpenter, B., Gelman, A., Hoffman, M., Lee, D., Goodrich, B., Betancourt, M., Brubaker, M. A., Guo, J., Li, P., and Riddell, A.: Stan: A probabilistic programming language, J. Stat. Softw., 20, 1-37, 2016.

Chiodo, G., Marsh, D. R., Garcia-Herrera, R., Calvo, N., and García, J. A.: On the detection of the solar signal in the tropical stratosphere, Atmos. Chem. Phys., 14, 5251-5269, https://doi.org/10.5194/acp-14-5251-2014, 2014.
Cox, R. T.: Probability, frequency, and reasonable expectation, Am. J. Phys., 14, 1-13, 1946.

Crutzen, P. J.: Ozone production rates in an oxygen-hydrogennitrogen oxide atmosphere, J. Geophys. Res., 76, 7311, https://doi.org/10.1029/JC076i030p07311, 1971.

Damadeo, R. P., Zawodny, J. M., Thomason, L. W., and Iyer, N.: SAGE version 7.0 algorithm: application to SAGE II, Atmos. Meas. Tech., 6, 3539-3561, https://doi.org/10.5194/amt-6-35392013, 2013.

Davis, S. M., Rosenlof, K. H., Hassler, B., Hurst, D. F., Read, W. G., Vömel, H., Selkirk, H., Fujiwara, M., and Damadeo, R.: The Stratospheric Water and Ozone Satellite Homogenized (SWOOSH) database: a long-term database for climate studies, Earth Syst. Sci. Data, 8, 461-490, https://doi.org/10.5194/essd8-461-2016, 2016.

DeLand, M. T., Taylor, S. L., Huang, L. K., and Fisher, B. L.: Calibration of the SBUV version 8.6 ozone data product, Atmos. Meas. Tech., 5, 2951-2967, https://doi.org/10.5194/amt-5-29512012, 2012.

Dudok de Wit, T., Bruinsma, S., and Shibasaki, K.: Synoptic radio observations as proxies for upper atmosphere modelling, Journal of Space Weather and Space Climate, 4, A06, https://doi.org/10.1051/swsc/2014003, 2014.

Frith, S. M., Kramarova, N. A., Stolarski, R. S., McPeters, R. D., Bhartia, P. K., and Labow, G. J.: Recent changes in total column ozone based on the SBUV Version 8.6 Merged Ozone Data Set, J. Geophys. Res.-Atmos., 119, 9735-9751, https://doi.org/10.1002/2014JD021889, 2014.

Froidevaux, L., Anderson, J., Wang, H.-J., Fuller, R. A., Schwartz, M. J., Santee, M. L., Livesey, N. J., Pumphrey, H. C., Bernath, P. F., Russell III, J. M., and McCormick, M. P.: Global OZone Chemistry And Related trace gas Data records for the Stratosphere (GOZCARDS): methodology and sample results with a focus on $\mathrm{HCl}, \mathrm{H}_{2} \mathrm{O}$, and $\mathrm{O}_{3}$, Atmos. Chem. Phys., 15, 1047110507, https://doi.org/10.5194/acp-15-10471-2015, 2015.

Funke, B., Baumgaertner, A., Calisto, M., Egorova, T., Jackman, C. H., Kieser, J., Krivolutsky, A., López-Puertas, M., Marsh, D. R., Reddmann, T., Rozanov, E., Salmi, S.-M., Sinnhuber, M., Stiller, G. P., Verronen, P. T., Versick, S., von Clarmann, T., Vyushkova, T. Y., Wieters, N., and Wissing, J. M.: Composition changes after the "Halloween" solar proton event: the High Energy Particle Precipitation in the Atmosphere (HEPPA) model versus MIPAS data intercomparison study, Atmos. Chem. Phys., 11, 90899139, https://doi.org/10.5194/acp-11-9089-2011, 2011.

Haigh, J. D.: The Role of Stratospheric Ozone in Modulating the Solar Radiative Forcing of Climate, Nature, 370, 544-546, https://doi.org/10.1038/370544a0, 1994.

Harris, N. R. P., Hassler, B., Tummon, F., Bodeker, G. E., Hubert, D., Petropavlovskikh, I., Steinbrecht, W., Anderson, J., Bhartia, P. K., Boone, C. D., Bourassa, A., Davis, S. M., Degenstein, D., Delcloo, A., Frith, S. M., Froidevaux, L., Godin-Beekmann, S., Jones, N., Kurylo, M. J., Kyrölä, E., Laine, M., Leblanc, S. T., Lambert, J.-C., Liley, B., Mahieu, E., Maycock, A., de Mazière, M., Parrish, A., Querel, R., Rosenlof, K. H., Roth, C., Sioris, C., Staehelin, J., Stolarski, R. S., Stübi, R., Tamminen, J., Vigouroux, C., Walker, K. A., Wang, H. J., Wild, J., and Zawodny, J. M.: Past changes in the vertical distribution of ozone - Part 3: Analysis and interpretation of trends, At- 
mos. Chem. Phys., 15, 9965-9982, https://doi.org/10.5194/acp15-9965-2015, 2015.

Hassler, B., Petropavlovskikh, I., Staehelin, J., August, T., Bhartia, P. K., Clerbaux, C., Degenstein, D., Mazière, M. D., Dinelli, B. M., Dudhia, A., Dufour, G., Frith, S. M., Froidevaux, L., GodinBeekmann, S., Granville, J., Harris, N. R. P., Hoppel, K., Hubert, D., Kasai, Y., Kurylo, M. J., Kyrölä, E., Lambert, J.-C., Levelt, P. F., McElroy, C. T., McPeters, R. D., Munro, R., Nakajima, H., Parrish, A., Raspollini, P., Remsberg, E. E., Rosenlof, K. H., Rozanov, A., Sano, T., Sasano, Y., Shiotani, M., Smit, H. G. J., Stiller, G., Tamminen, J., Tarasick, D. W., Urban, J., van der A, R. J., Veefkind, J. P., Vigouroux, C., von Clarmann, T., von Savigny, C., Walker, K. A., Weber, M., Wild, J., and Zawodny, J. M.: Past changes in the vertical distribution of ozone - Part 1 : Measurement techniques, uncertainties and availability, Atmos. Meas. Tech., 7, 1395-1427, https://doi.org/10.5194/amt-7-13952014, 2014.

Hubert, D., Lambert, J.-C., Verhoelst, T., Granville, J., Keppens, A., Baray, J.-L., Bourassa, A. E., Cortesi, U., Degenstein, D. A., Froidevaux, L., Godin-Beekmann, S., Hoppel, K. W., Johnson, B. J., Kyrölä, E., Leblanc, T., Lichtenberg, G., Marchand, M., McElroy, C. T., Murtagh, D., Nakane, H., Portafaix, T., Querel, R., Russell III, J. M., Salvador, J., Smit, H. G. J., Stebel, K., Steinbrecht, W., Strawbridge, K. B., Stübi, R., Swart, D. P. J., Taha, G., Tarasick, D. W., Thompson, A. M., Urban, J., van Gijsel, J. A. E., Van Malderen, R., von der Gathen, P., Walker, K. A., Wolfram, E., and Zawodny, J. M.: Ground-based assessment of the bias and long-term stability of 14 limb and occultation ozone profile data records, Atmos. Meas. Tech., 9, 2497-2534, https://doi.org/10.5194/amt-9-2497-2016, 2016.

Johnston, H.: Reduction of Stratospheric Ozone by Nitrogen Oxide Catalysts from Supersonic Transport Exhaust, Science, 173, 517-522, https://doi.org/10.1126/science.173.3996.517, 1971.

Kidston, J., Scaife, A. A., Hardiman, S. C., Mitchell, D. M., Butchart, N., Baldwin, M. P., and Gray, L. J.: Stratospheric influence on tropospheric jet streams, storm tracks and surface weather, Nat. Geosci., 8, 433-440, https://doi.org/10.1038/ngeo2424, 2015.

Kirgis, G., Leblanc, T., McDermid, I. S., and Walsh, T. D.: Stratospheric ozone interannual variability (1995-2011) as observed by lidar and satellite at Mauna Loa Observatory, HI and Table Mountain Facility, CA, Atmos. Chem. Phys., 13, 5033-5047, https://doi.org/10.5194/acp-13-5033-2013, 2013.

Kramarova, N. A., Bhartia, P. K., Frith, S. M., McPeters, R. D., and Stolarski, R. S.: Interpreting SBUV smoothing errors: an example using the quasi-biennial oscillation, Atmos. Meas. Tech., 6, 2089-2099, https://doi.org/10.5194/amt-6-2089-2013, $2013 \mathrm{a}$.

Kramarova, N. A., Frith, S. M., Bhartia, P. K., McPeters, R. D., Taylor, S. L., Fisher, B. L., Labow, G. J., and DeLand, M. T.: Validation of ozone monthly zonal mean profiles obtained from the version 8.6 Solar Backscatter Ultraviolet algorithm, Atmos. Chem. Phys., 13, 6887-6905, https://doi.org/10.5194/acp13-6887-2013, 2013b.

Krueger, A. J., Guenther, B., Fleig, A. J., Heath, D. F., Hilsenrath, E., McPeters, R., and Prabhakara, C.: Satellite ozone measurements, Philos. T. R. Soc.-S. A, 296, 191-204, https://doi.org/10.1098/rsta.1980.0164, 1980.

Kuchar, A., Sacha, P., Miksovsky, J., and Pisoft, P.: The 11-year solar cycle in current reanalyses: a (non)linear attribution study of the middle atmosphere, Atmos. Chem. Phys., 15, 6879-6895, https://doi.org/10.5194/acp-15-6879-2015, 2015.

Kyrölä, E., Laine, M., Sofieva, V., Tamminen, J., Päivärinta, S.M., Tukiainen, S., Zawodny, J., and Thomason, L.: Combined SAGE II-GOMOS ozone profile data set for 1984-2011 and trend analysis of the vertical distribution of ozone, Atmos. Chem. Phys., 13, 10645-10658, https://doi.org/10.5194/acp-13-106452013, 2013.

Labow, G. J., McPeters, R. D., Bhartia, P. K., and Kramarova, N.: A comparison of 40 years of SBUV measurements of column ozone with data from the Dobson/Brewer network, J. Geophys. Res.Atmos., 118, 7370-7378, https://doi.org/10.1002/jgrd.50503, 2013.

Laine, M., Latva-Pukkila, N., and Kyrölä, E.: Analysing timevarying trends in stratospheric ozone time series using the state space approach, Atmos. Chem. Phys., 14, 9707-9725, https://doi.org/10.5194/acp-14-9707-2014, 2014.

Lee, T. C. K., Zwiers, F. W., Hegerl, G. C., Zhang, X., and Tsao, M.: A Bayesian Climate Change Detection and Attribution Assessment., J. Climate, 18, 2429-2440, https://doi.org/10.1175/JCLI3402.1, 2005.

Maycock, A. C., Matthes, K., Tegtmeier, S., Thiéblemont, R., and Hood, L.: The representation of solar cycle signals in stratospheric ozone - Part 1: A comparison of recently updated satellite observations, Atmos. Chem. Phys., 16, 10021-10043, https://doi.org/10.5194/acp-16-10021-2016, 2016.

McLinden, C. A., Tegtmeier, S., and Fioletov, V.: Technical Note: A SAGE-corrected SBUV zonal-mean ozone data set, Atmos. Chem. Phys., 9, 7963-7972, https://doi.org/10.5194/acp-9-79632009, 2009.

McPeters, R. D., Bhartia, P. K., Haffner, D., Labow, G. J., and Flynn, L.: The version 8.6 SBUV ozone data record: An overview, J. Geophys. Res.-Atmos., 118, 8032-8039, https://doi.org/10.1002/jgrd.50597, 2013.

Mironova, I. A., Aplin, K. L., Arnold, F., Bazilevskaya, G. A., Harrison, R. G., Krivolutsky, A. A., Nicoll, K. A., Rozanov, E. V., Turunen, E., and Usoskin, I. G.: Energetic Particle Influence on the Earth's Atmosphere, Space Sci. Rev., 194, 1-96, https://doi.org/10.1007/s11214-015-0185-4, 2015.

Molina, M. J. and Rowland, F. S.: Stratospheric sink for chlorofluoromethanes: chlorine atomc-atalysed destruction of ozone, Nature, 249, 810-812, https://doi.org/10.1038/249810a0, 1974.

Nair, P. J., Godin-Beekmann, S., Froidevaux, L., Flynn, L. E., Zawodny, J. M., Russell III, J. M., Pazmiño, A., Ancellet, G., Steinbrecht, W., Claude, H., Leblanc, T., McDermid, S., van Gijsel, J. A. E., Johnson, B., Thomas, A., Hubert, D., Lambert, J.-C., Nakane, H., and Swart, D. P. J.: Relative drifts and stability of satellite and ground-based stratospheric ozone profiles at NDACC lidar stations, Atmos. Meas. Tech., 5, 1301-1318, https://doi.org/10.5194/amt-5-1301-2012, 2012.

Nazaryan, H. and McCormick, M. P.: Comparisons of Stratospheric Aerosol and Gas Experiment (SAGE II) and Solar Backscatter Ultraviolet Instrument (SBUV/2) ozone profiles and trend estimates, J. Geophys. Res.-Atmos., 110, D17302, https://doi.org/10.1029/2004JD005483, 2005.

Neal, R. M.: Probabilistic inference using Markov chain Monte Carlo methods, Technical Report, CRG-TR-93-1, University of Toronto, Toronto, 1993. 
Newman, P. A., Nash, E. R., and Rosenfield, J. E.: What controls the temperature of the Arctic stratosphere during the spring?, J. Geophys. Res., 106, 19999-20010, https://doi.org/10.1029/2000JD000061, 2001.

Polvani, L. M., Waugh, D. W., Correa, G. J. P., and Son, S.-W.: Stratospheric Ozone Depletion: The Main Driver of Twentieth-Century Atmospheric Circulation Changes in the Southern Hemisphere, J. Climate, 24, 795-812, https://doi.org/10.1175/2010JCLI3772.1, 2011.

Robock, A.: Volcanic eruptions and climate, Rev. Geophys., 38, 191-219, https://doi.org/10.1029/1998RG000054, 2000.

Sato, M., Hansen, J. E., McCormick, M. P., and Pollack, J. B.: Stratospheric aerosol optical depths, 18501990, J. Geophys. Res.-Atmos., 98, 22987-22994, https://doi.org/10.1029/93JD02553, 1993.

Shepherd, T. G., Plummer, D. A., Scinocca, J. F., Hegglin, M. I., Fioletov, V. E., Reader, M. C., Remsberg, E., von Clarmann, T., and Wang, H. J.: Reconciliation of halogen-induced ozone loss with the total-column ozone record, Nat. Geosci., 7, 443-449, https://doi.org/10.1038/ngeo2155, 2014.

Sioris, C. E., McLinden, C. A., Fioletov, V. E., Adams, C., Zawodny, J. M., Bourassa, A. E., Roth, C. Z., and Degenstein, D. A.: Trend and variability in ozone in the tropical lower stratosphere over 2.5 solar cycles observed by SAGE II and OSIRIS, Atmos. Chem. Phys., 14, 3479-3496, https://doi.org/10.5194/acp-14-3479-2014, 2014.

Sofieva, V. F., Rahpoe, N., Tamminen, J., Kyrölä, E., Kalakoski, N., Weber, M., Rozanov, A., von Savigny, C., Laeng, A., von Clarmann, T., Stiller, G., Lossow, S., Degenstein, D., Bourassa, A., Adams, C., Roth, C., Lloyd, N., Bernath, P., Hargreaves, R. J., Urban, J., Murtagh, D., Hauchecorne, A., Dalaudier, F., van Roozendael, M., Kalb, N., and Zehner, C.: Harmonized dataset of ozone profiles from satellite limb and occultation measurements, Earth Syst. Sci. Data, 5, 349-363, https://doi.org/10.5194/essd5-349-2013, 2013.

Sofieva, V. F., Kalakoski, N., Päivärinta, S.-M., Tamminen, J., Laine, M., and Froidevaux, L.: On sampling uncertainty of satellite ozone profile measurements, Atmos. Meas. Tech., 7, 18911900, https://doi.org/10.5194/amt-7-1891-2014, 2014.

Solomon, S., Ivy, D. J., Kinnison, D., Mills, M. J., Neely, R. R., and Schmidt, A.: Emergence of healing in the Antarctic ozone layer, Science, 353, 269-274, https://doi.org/10.1126/science.aae0061, 2016.
Soukharev, B. E. and Hood, L. L.: Solar cycle variation of stratospheric ozone: Multiple regression analysis of long-term satellite data sets and comparisons with models, J. Geophys. Res.Atmos., 111, D20314, https://doi.org/10.1029/2006JD007107, 2006.

Staehelin, J., Renaud, A., Bader, J., McPeters, R., Viatte, P., Hoegger, B., Bugnion, V., Giroud, M., and Schill, H.: Total ozone series at Arosa (Switzerland): Homogenization and data comparison, J. Geophys. Res., 103, 5827-5841, https://doi.org/10.1029/97JD02402, 1998.

Tiao, G. C., Xu, D., Pedrick, J. H., Zhu, X., and Reinsel, G. C.: Effects of autocorrelation and temporal sampling schemes on estimates of trend and spatial correlation, J. Geophys. Res., 95, 20507-20517, https://doi.org/10.1029/JD095iD12p20507, 1990.

Toohey, M., Hegglin, M. I., Tegtmeier, S., Anderson, J., Añel, J. A., Bourassa, A., Brohede, S., Degenstein, D., Froidevaux, L., Fuller, R., Funke, B., Gille, J., Jones, A., Kasai, Y., Krüger, K., Kyrölä, E., Neu, J. L., Rozanov, A., Smith, L., Urban, J., Clarmann, T., Walker, K. A., and Wang, R. H. J.: Characterizing sampling biases in the trace gas climatologies of the SPARC Data Initiative, J. Geophys. Res.-Atmos., 118, 11847-11862, https://doi.org/10.1002/jgrd.50874, 2013.

Tummon, F., Hassler, B., Harris, N. R. P., Staehelin, J., Steinbrecht, W., Anderson, J., Bodeker, G. E., Bourassa, A., Davis, S. M., Degenstein, D., Frith, S. M., Froidevaux, L., Kyrölä, E., Laine, M., Long, C., Penckwitt, A. A., Sioris, C. E., Rosenlof, K. H., Roth, C., Wang, H.-J., and Wild, J.: Intercomparison of vertically resolved merged satellite ozone data sets: interannual variability and long-term trends, Atmos. Chem. Phys., 15, 3021-3043, https://doi.org/10.5194/acp-15-3021-2015, 2015.

Wang, H. J., Cunnold, D. M., Thomason, L. W., Zawodny, J. M., and Bodeker, G. E.: Assessment of SAGE version 6.1 ozone data quality, J. Geophys. Res.-Atmos., 107, 4691, https://doi.org/10.1029/2002JD002418, 2002.

Wild, J. D. and Long, C. S.: A Coherent Ozone Profile Dataset from SBUV, SBUV/2: 1979 to 2016, in preparation, 2017.

WMO: Scientific Assessment of Ozone Depletion: 2010, Global Ozone Research and Monitoring Project, 52, 516, 2011.

WMO: Scientific Assessment of Ozone Depletion: 2014 Global Ozone Research and Monitoring Project Report, World Meteorological Organization, Geneva, Switzerland, p. 416, 2014.

WMO/UNEP: Scientific Assessment of Ozone Depletion: 1994, World Meteorological Organization, Geneva, Switzerland, 1994. 